\title{
Prediction of Turbulent Temperature Fluctuations in Hot Jets
}

\author{
James R. DeBonis* \\ NASA Glenn Research Center, Cleveland, Ohio 44135
}

\begin{abstract}
Large-eddy simulations (LES) were used to investigate turbulent temperature fluctuations and turbulent heat flux in hot jets. A high-resolution finite-difference Navier-Stokes solver was used to compute the flow from a 2-inch round nozzle. Three different flow conditions of varying jet Mach numbers and temperature ratios were examined. The LES results showed that the temperature field behaves similar to the velocity field, but with a more rapidly spreading mixing layer. Predictions of mean, $\bar{u}_{i}$, and fluctuating, $u_{i}^{\prime}$, velocities were compared to particle image velocimetry data. Predictions of mean, $\bar{T}$, and fluctuating, $T^{\prime}$, temperature were compared to data obtained using Rayleigh scattering and Raman spectroscopy. Very good agreement with experimental data was demonstrated for the mean and fluctuating velocities. The LES correctly predicts the behavior of the turbulent temperature field, but over-predicts the levels of the fluctuations. The turbulent heat flux was examined and compared to Reynold-averaged Navier-Stokes (RANS) results. The LES and RANS simulations produced very similar results for the radial heat flux. However, the axial heat flux obtained from the LES differed significantly from the RANS result in both structure and magnitude, indicating that the gradient diffusion type model in RANS is inadequate. Finally, the LES data was used to compute the turbulent Prandtl number and verify that a constant value of 0.7 used in the RANS models is a reasonable assumption.
\end{abstract}

\section{Nomenclature}

$\begin{array}{ll}c_{p} & \text { specific heat at constant pressure } \\ e_{t} & \text { total energy } \\ k & \text { turbulent kinetic energy } \\ p & \text { pressure } \\ q_{i} & \text { heat flux vector } \\ q_{x}^{T}, q_{r}^{T} & \text { axial \& radial components of the turbulent heat flux vector } \\ t & \text { time } \\ u_{i} & \text { velocity vector } \\ x_{i} & \text { coordinate vector } \\ x, r & \text { axial and radial coordinates } \\ D & \text { diameter } \\ M & \text { Mach number } \\ \mathrm{NPR} & \text { nozzle pressure ratio } \\ P r & \text { Prandtl number } \\ P r_{t} & \text { turbulent Prandt number } \\ S_{i j} & \text { strain rate tensor } \\ T & \text { temperature } \\ U_{j} & \text { jet exit velocity } \\ \Delta T_{j} & \text { temperature difference, } \Delta T_{j}=T_{j}-T_{\infty} \\ \delta_{i j} & \text { Kronecker delta } \\ \epsilon_{M} & \text { diffusivity of momemtum } \\ \end{array}$

*Aerospace Engineer, Inlets and Nozzles Branch, james.r.debonis@nasa.gov, Associate Fellow AIAA 


$\begin{array}{ll}\epsilon_{T} & \text { diffusivity of temperature } \\ \mu & \text { kinematic viscosity } \\ \rho & \text { density } \\ \tau_{i j} & \text { stress tensor }\end{array}$

$\begin{array}{ll}\text { subscripts } & \\ a & \text { acoustic } \\ j & \text { jet } \\ \infty & \text { freestream condition }\end{array}$

$\begin{array}{ll}\text { superscripts } & \\ - & \text { fluctuating quantity } \\ & \text { time averaged quantity } \\ & \text { density weighted, time averaged quantity } \\ T & \text { turbulent }\end{array}$

\section{Introduction}

Accurate prediction of turbulent fluid flows remains one of the key challenges in fluid dynamics. The most often used approach for computing turbulent flows is solving the Reynolds-averaged Navier-Stokes (RANS) equations and associated turbulence model equation(s). This approach yields good results for attached flows, but typically fails for separated flows and free shear flows. Scale resolving simulations, large-eddy simulation (LES) and direct numerical simulation (DNS), offer improved accuracy, but at a significant increase in simulation time and cost. For this reason, RANS approaches are envisioned to remain the primary solution technique for years to come. ${ }^{1}$ LES and DNS will be reserved for cases where their increased accuracy is needed and cost is not an issue.

The Reynolds-averaged Navier-Stokes equations have undetermined terms in both the momentum and energy equations that must be "closed" with a model. The turbulence model is typically developed for the unclosed momentum terms, the turbulent Reynolds stresses. These models vary in complexity from simple algebraic relations, to seven equation full Reynolds stress models. The vast majority of analyses employ one- and two-equation transport models. The unclosed term for the energy equation is the turbulent heat flux vector. Like the the turbulent Reynolds stresses, there are varying levels of complexity in the modeling of this quantity. The simplest approach is using the gradient diffusion assumption and Reynolds analogy; using a constant value for the turbulent Prandtl number. Approaches increasing in complexity include variable Prandtl number models, scalar variance models, and scalar flux models. The scalar variance models solve transport equations for the variance of a thermal quantity, such as temperature, and are analogous to two-equation turbulence models. The scalar flux models solve for the components of the turbulent heat flux vector and are analogous to the full Reynolds stress models. Unlike the momentum equation, the vast majority of analyses still rely on the simplistic gradient diffusion approach. This is largely due to the limited amount of experimental data for the turbulent heat flux, necessary to develop and validate new models.

Measurements of the heat flux vector require simultaneous time-resolved measurements of velocity, temperature and density (for compressible flows). Much of the available data has been obtained for incompressible flows through a combination of hot wire anemometry and cold wire thermometry. ${ }^{2-5}$ The probes for these measurements are intrusive and must contain three wires to obtain two velocity components and temperature; this results in a large measurement volume. Hot/cold wire measurements are also difficult in high speed/high temperature flows due to wire breakage and stretching; ${ }^{6}$ temperature measurements are especially problematic. ${ }^{7}$ Accurate non-intrusive laser-based measurements of velocity using laser Doppler velocimetry $(\mathrm{LDV})^{8}$ and particle image velocimetry (PIV $)^{9}$ are commonplace in high-speed and high-temperature flows. Several laser-based temperature measurements have been demonstrated ${ }^{10-14}$ but have not reached the level of maturity of LDV and PIV. Accurate non-intrusive simultaneous measurement of velocity and temperature has not been demonstrated in large-scale test facilities.

Round jets are an example of a free shear flow where RANS methods fail to accurately predict the flow physics. ${ }^{15,16}$ The effects of compressibility and heating are both poorly represented by the standard RANS models. For this flow LES has already been shown to accurately predict both the mean velocities and turbulence intensities at a variety of conditions. ${ }^{17-19}$ For this reason, the heated round jet is a good 
candidate to explore the possibility of using large-eddy simulation to examine the deficiencies in the RANS modeling of the turbulent heat flux for a compressible high temperature flow, where simultaneous velocity and temperature measurements are difficult.

The work presented herein seeks to examine the fluctuating temperature fields and the turbulent heat flux vector in turbulent jets using large-eddy simulation. RANS and LES simulations were carried out on a 2-inch round jet at three different flow conditions; low-speed cold jet, low-speed hot jet, and high-speed hot jet. The computations were validated by comparing with experimental data from three sources: 1) velocity data using particle image velocimetry, ${ }^{20} 2$ ) temperature measurements using Rayleigh scattering, ${ }^{21}$ and 3) newly obtained temperature measurements using Raman spectroscopy. ${ }^{22}$ Successful validation of the individual fluctuating velocities and temperatures, $u^{\prime}$ and $T^{\prime}$ will lend credence to the LES obtained turbulent heat flux vector, $\overline{\rho u^{\prime} T^{\prime}}$.

\section{Reynolds-Averaged Navier-Stokes Equations}

The vast majority of computational fluid dynamic (CFD) simulations are RANS simulations. These simulations produce a time-averaged flow field using a turbulence model. The Reynolds-averaged NavierStokes equations, in compressible form, are derived from the standard form of the equations by a density weighted time averaging procedure.

$$
\hat{u}_{i}=\frac{1}{\bar{\rho} \tau} \int_{t}^{t+\tau} \rho u_{i} d t
$$

The instantaneous variables are decomposed into a mean and fluctuating part. For example, velocity is decomposed as $u_{i}=\hat{u}_{i}+u_{i}^{\prime}$. The resulting equations for the conservation of continuity, momentum and energy are given in equations 2,3 and 4 .

$$
\begin{gathered}
\frac{\partial \bar{\rho}}{\partial t}+\frac{\partial}{\partial x_{i}}\left(\bar{\rho} \hat{u}_{i}\right)=0 \\
\frac{\partial}{\partial t}\left(\bar{\rho} \hat{u}_{i}\right)+\frac{\partial}{\partial x_{j}}\left(\bar{\rho} \hat{u}_{i} \hat{u}_{j}\right)+\frac{\partial \bar{p}}{\partial x_{i}}-\frac{\partial \bar{\tau}_{i j}}{\partial x_{j}}+\frac{\partial}{\partial x_{j}}\left(\overline{\rho u_{i}^{\prime} u_{j}^{\prime}}\right)=0 \\
\frac{\partial}{\partial t}\left(\bar{\rho} \hat{e}_{t}\right)+\frac{\partial}{\partial x_{j}}\left(\bar{\rho} \hat{u}_{j} \hat{e}_{t}+\hat{u}_{j} \bar{p}\right)-\frac{\partial}{\partial x_{j}}\left[\hat{u}_{i} \bar{\tau}_{i j}-\hat{u}_{i}\left(\overline{\rho u_{i}^{\prime} u_{j}^{\prime}}\right)\right]+\frac{\partial}{\partial x_{j}}\left(\bar{q}_{j}+c_{p} \overline{\rho u_{j}^{\prime} T^{\prime}}\right)=0
\end{gathered}
$$

where

$$
\bar{\tau}_{i j}=2 \mu\left[\frac{1}{2}\left(\frac{\partial \hat{u}_{i}}{\partial x_{j}}+\frac{\partial \hat{u}_{j}}{\partial x_{i}}\right)-\frac{1}{3} \frac{\partial \hat{u}_{k}}{\partial x_{k}} \delta_{i j}\right]
$$

and

$$
\bar{q}_{j}=-c_{p} \frac{\mu}{\operatorname{Pr}} \frac{\partial \bar{T}}{\partial x_{j}}
$$

The equations are expressed in terms of time averaged variables, with the exception of two unclosed terms that must be modeled. The first, $\tau_{i j}^{T}=\overline{\rho u_{i}^{\prime} u_{j}^{\prime}}$ is the Reynolds stress tensor (equations $3 \& 4$ ). The second is the turbulent heat flux vector $q_{j}^{T}=c_{p} \overline{\rho u_{j}^{\prime} T^{\prime}}$, (equation 4 ).

By far, the most common method to close the equations is through the use of linear eddy viscosity models. In this approach a linear relationship between the Reynolds stress and mean strain tensors is assumed,

$$
-\overline{\rho u_{i}^{\prime} u_{j}^{\prime}}=\mu_{t}\left(2 \hat{S}_{i j}-\frac{2}{3} \frac{\partial \hat{u}_{k}}{\partial x_{k}} \delta_{i j}\right)-\frac{2}{3} \bar{\rho} k \delta_{i j}
$$

where

$$
\hat{S}_{i j}=\frac{1}{2}\left(\frac{\partial \hat{u}_{i}}{\partial x_{j}}+\frac{\partial \hat{u}_{j}}{\partial x_{i}}\right)
$$

is the mean strain rate tensor. The eddy viscosity, $\mu_{t}$, in this relationship is computed by the turbulence model. The most widely used models are the Shear Stress Transport (SST), ${ }^{23}$ Spalart-Allmaras (SA) ${ }^{24}$ and $k-\epsilon^{25}$ models.

The Reynolds stress tensor and mean strain rates are routinely measured in experiments by a number of methods including hot-wire anemometry, laser-doppler velocimetry (LDV), and particle image velocimetry 
(PIV). As such, there are extensive datasets available to aid in developing and validating models for the Reynolds stresses.

In contrast, the turbulent heat flux vector has proven more difficult to measure and datasets are fewer. As a result, most turbulent simulations rely on the simplistic Reynolds analogy to relate the turbulent heat transfer to the turbulent momentum transfer. Thus the turbulent heat flux vector is modeled using the gradient diffusion assumption.

$$
c_{p} \overline{\rho u_{j}^{\prime} T^{\prime}}=-c_{p} \frac{\mu_{t}}{P r_{t}} \frac{\partial \bar{T}}{\partial x_{j}}
$$

where the turbulent heat flux vector is directly related to the temperature gradient by the eddy viscosity, $\mu_{t}$, and the turbulent Prandtl nuber, $P r_{t}$. The turbulent Prandtl number is typically taken to be a constant. However, there are several models that compute $P r_{t}$ as a function of the flow. Yoder provides a good overview of these models and their application to jet flows. ${ }^{26}$

\section{Experimental Data}

Researchers at NASA's Glenn Research Center have performed extensive measurements of the flow fields from a series of subsonic round jets during several test campaigns. Two different, 2-inch round convergent nozzle geometries were used for these tests: the Acoustic Reference Nozzle (ARN2) and the Small Metal Chevron (SMC000) nozzle. Flow conditions, based on the acoustic Mach number, $M_{a}=U_{j} / \sqrt{\gamma R T_{\infty}}$, and static temperature ratio, $T_{j} / T_{\infty}$, were chosen to replicate the set of flow conditions known as the Tanna matrix. ${ }^{27}$ These tests were all carried out on the Small Hot Jet Acoustic Rig (SHJAR) in the Aeroacoustic Propulsion Laboratory (AAPL). The SHJAR can provide 150 psi air at mass flow rates up to $6 \mathrm{lbm} / \mathrm{s}$ and temperatures up to $1300^{\circ} \mathrm{F}$. Three datasets are used in this study: velocity measurements using PIV, temperature measurements using Rayleigh scattering, and temperature measurements using Raman spectroscopy. It must be noted that while PIV is a well established technique for velocimetry, the temperature measurements are of an exploratory nature; hence they contain more uncertainty and do not have historical data for comparison.

\section{A. Particle Image Velocimetry}

Bridges and Wernet conducted extensive velocity field measurements using two- and three-component particle image velocimetry of jet flow fields from both the ARN2 and SMC000 nozzles over several different test campaigns. The data from these different tests were combined into a single consensus dataset used here. ${ }^{20}$ This data compares well with other data in the literature, and has been used extensively for CFD validation. The data contains the mean and variances of all three velocity components for 7 sets of flow conditions (referred to as set points) in the Tanna Matrix. Uncertainties in the data are provided and quoted as 2 to 4 percent in the mean velocity, $u / U_{j}$, and 7 to 14 percent in axial turbulence intensity, $u^{\prime} / U_{j}$.

\section{B. Rayleigh Scattering}

Mielke performed time resolved velocity, temperature and density measurements in the flow fields from the SMC000 nozzle on the SHJAR using Rayleigh scattering. ${ }^{21}$ Sampling rates up to $32 \mathrm{kHz}$ were used. Data were obtained for axial profiles on the jet centerline and lipline, and radial profiles at $x / D_{j}=2,3,5,6,7$ and 10. The Rayleigh scattering technique requires clean air flow and is very sensitive to particle contamination. A low-speed co-flow system was set up around the SHJAR nozzle to provide particulate free air. The system was powered by a blower and particulates were removed by sub-micron HEPA filters. Despite these efforts some contamination occurred; mostly at downstream locations where ambient air was entrained into the jet plume. Mielke reports rms error ranging from \pm 0.2 to \pm 0.5 of $\Delta T_{j}$. Error in $T^{\prime} / \Delta T_{j}$ was a function of the shot noise and was shown to be up to $0.043 \Delta T_{j}$ or about $20 \%$ of the peak value.

\section{Raman Spectroscopy}

Locke and Wernet recently used Raman spectroscopy to measure instantaneous temperature in the flow fields from the SMC000 nozzle. ${ }^{22}$ Raman spectroscopy was chosen as it was deemed less sensitive to the environmental conditions of the test, namely: large temperature variations, high sonic noise levels, high ambient light levels, and inaccessibility during the test. Both mean and fluctuating temperature measurements were 
obtained for axial profiles on the jet centerline and lipline, and radial profiles at $x / D_{j}=2,4,8,12$ and 20. Error estimates based on calibration data for mean temperature were less than $2.5 \%$. The experimentalist also noted that additional spatial averaging of the data may have occurred due to the amount of flow advecting through the probe volume during the $500 \mathrm{nsec}$ laser pulse. The effect of the length of the laser pulse on $T^{\prime} / \Delta T_{j}$ measurements will be examined in future testing.

\section{Computations}

\section{A. Large-Eddy Simulations}

To bridge the gap between individually measured/uncorrelated velocity and temperature data, and the turbulent heat flux vector, a series of large-eddy simulations were carried out. The LES will be validated against both the velocity and temperature data for several flow conditions. Satisfactory validation of the fluctuating velocity and temperatures will provide confidence that the correlated quantity $\overline{\rho u^{\prime} T^{\prime}}$ is correctly predicted. The combination of experimental data and validated LES data can then serve as a basis for heat flux model development and validation.

\section{Code Description}

The code used in this study, WRLES (Wave Resolving Large-Eddy Simulation), is a special-purpose largeeddy simulation code that uses high-resolution temporal and spatial discretization schemes to accurately simulate the convection of turbulent structures. The code solves the compressible Favre-filtered Navier-Stokes equations on structured meshes using generalized curvilinear coordinates. WRLES has been previously used and validated for jet flows. ${ }^{17,28-30}$

WRLES employs finite-difference methods to solve the equations. Central-differencing operators up to 12th-order accuracy are implemented for the spatial discretization. Solution filtering is used to provide stability with minimal dissipation. Several $2 \mathrm{~N}$-storage Runge-Kutta schemes are implemented for temporal discretization.

For this work, the 11-point dispersion relation preserving scheme, and matching solution filter of Bogey and Bailley were used for the spatial discretization. ${ }^{31}$ Carpenter and Kennedy's 4-stage, 3rd order RungeKutta scheme was used for time advancement. ${ }^{32}$ No explicit sub-grid scale model was used, and hence this can be classified as an implicit LES approach.

\section{Computational Grid}

The grids used for the data presented in this study model the 2-inch diameter acoustic reference nozzle (ARN2), one of two models used for the PIV database. The LES grid was generated using the Pointwise software $^{33}$ and uses an o-grid topology in the cross-section and contains 36 million grid points. The region encompassing the jet plume contains 912 axial points, 184 radial points, and 181 evenly spaced azimuthal points (every 2 degrees). The computational domain extends 30 jet diameters downstream of the nozzle exit and 30 jet diameters outward from the centerline.

The grid is designed to accurately resolve the flow for the first 15 jet diameters downstream of the nozzle exit. In this region there are 864 axial grid points. A very low stretching rate was prescribed for the first 10 diameters. Beyond this point, the stretching rate increases more rapidly. The very large grid spacings at the downstream portion of the domain are designed to dissipate outgoing waves so that they do not reflect off of the boundary and reenter the domain. At the nozzle exit the radial spacing is smallest at the nozzle

lip $\left(r / D_{j}=0.5\right)$ and this spacing increases with axial distance. Figure 1 shows the grid spacings in both the axial and radial directions.

\section{Boundary Conditions}

Total pressures and total temperatures based on the prescribed jet conditions (Table 1) were specified at the inflow plane of the nozzle plenum. Ambient static pressure was specified at the outflow plane. Farfield characteristic boundaries were applied to the outer boundaries. No-slip adiabatic conditions were applied to the solid surfaces. A special differencing technique was used to span the singularity on the grid's centerline. Details of this method can be found in a previous paper. ${ }^{17}$ An additional filter, with a lower cutoff wave number (Kennedy \& Carpenter's 6th order filter ${ }^{34}$ ) was applied to the solution over the last 10 diameters 
to further prevent unwanted waves reflecting from the outflow boundary. No artificial inflow turbulence treatment was used and the boundary layer on the interior of the nozzle is thin and the flow is steady. The jet mixing layer transitions very quickly downstream of the nozzle exit.

Table 1: Flow conditions

\begin{tabular}{rrrrr} 
Set Point & $M_{a}$ & $T_{j} / T_{\infty}$ & $\mathrm{NPR}$ & $M_{j}$ \\
\hline 3 & 0.5 & 0.95 & 1.197 & 0.513 \\
23 & 0.5 & 1.764 & 1.102 & 0.376 \\
27 & 0.9 & 1.764 & 1.357 & 0.678
\end{tabular}

\section{B. Reynolds-Averaged Navier-Stokes Simulations}

Reynolds-averaged Navier-Stokes simulations were performed to provide representative RANS solutions for comparison to the LES and the experiment. The Shear Stress Transport model ${ }^{23}$ was used and resulting turbulent heat flux was extracted and compared to the LES derived values.

\section{Code Description}

The code used for the RANS simulations was Wind-US, a general purpose solver developed and distributed by the NPARC Alliance, a partnership between the NASA Glenn Research Center and the U.S. Air Force's Arnold Engineering Development Center. ${ }^{35}$ For these simulations, the structured grid flow solver was used in axisymmetric mode. The default flow solver settings were used. The explicit operator for the right-hand side is second-order upwind-biased for stretched grids. The implicit operator for the left-hand side is full block implicit. The SST turbulence model in Wind-US uses vorticity magnitude in the production term, ${ }^{36}$ which differs from the standard implementation of the model. The turbulent Prandtl number was assumed to be constant and a value of 0.7 was used.

\section{Computational Grid}

The computational grids used for the RANS calculations were taken from the NASA Langley Turbulence Modeling Resource website, turbmodels.larc.nasa.gov. Several grids of varying resolution are available. Grid convergence was achieved using the two-dimensional grid containing 73,151 points, and all results presented are from those calculations. The region encompassing the jet plume contains 257 axial points and 225 radial points.

\section{Boundary Conditions}

The RANS boundary conditions are a simpler subset of the LES computation. Total pressures and total temperatures based on the prescribed jet conditions (Table 1) were specified at the inflow plane of the nozzle plenum and the inflow plane of the ambient region. Ambient static pressure was specified at the outflow plane. A farfield characteristic boundary was applied to the outer boundary. No-slip adiabatic conditions were applied to the solid surfaces. Symmetry is enforced on the jet centerline.

\section{Results}

Three cases from the experimental test matrix were computed corresponding to set points (SP) 3, 23, and 27 (Table 1). Set points 3 and 23 represent a cold and hot jet at the same acoustic Mach number $\left(M_{a}=U_{j} / a_{\infty}\right)$ of 0.5 , and exit velocity of $558 \mathrm{ft} / \mathrm{s}$. Set point 27 represents a higher speed jet $\left(M_{a}=0.9\right)$ at the same temperature ratio as set point 23. The ambient conditions were set to standard atmospheric pressure and temperature. A small forward velocity, $M=0.05$, was used to maintain well-posed farfield boundary conditions.

A characteristic time unit is defined as $t^{*}=\left(t D_{j}\right) / U_{j}$. This represents the time it takes for a particle of fluid moving at the jet velocity to traverse one jet diameter. The solution was initialized to the ambient 
state and then run for approximately $60 t^{*}$ to establish a fully turbulent jet flow, free of initial transients. All calculations were then run for a minimum of $180 t^{*}$ to accrue data for time-averaging. Density, velocity and temperature, and moments of those quantities were averaged at every point. Turbulent statistics were computed from these quantities and then averaged over the azimuth to provide two-dimensional descriptions of the jets.

\section{A. Effect of Grid Resolution on LES Accuracy}

Adequate grid resolution is critical for accurate LES computations. As stated above, the current grid is designed to accurately resolve the first 15 jet diameters of the jet plume. This was verified by the following comparison. Figure 2 compares the axial turbulence intensity from the LES to the experimental data for SP 27 on the both the jet centerline $\left(r / D_{j}=0\right)$ and lipline $\left(r / D_{j}=0.5\right)$ for the entire axial domain. The experimental uncertainty is included in the plot. The axial grid spacing is plotted against the right-hand axis. The agreement with experiment is excellent for the first 10 diameters. Beyond this point, the grid spacing begins to increase more rapidly and the agreement between prediction and experiment begins to degrade. The LES data falls outside the uncertainty bounds of the data at $x / D_{j}=18$. All further data presented here will be limited to $x / D_{j} \leq 15$.

\section{B. Comparison to Velocity Data}

The LES and RANS solutions are compared to Bridges and Wernet's experimental PIV data ${ }^{20}$ in figures 3 , 4,5 , and 6. Data on the jet centerline, $r / D_{j}=0.0$, and the jet lipline, $r / D_{j}=0.5$, are plotted. The RANS simulations only directly produce $\bar{u} / U_{j}$ and $k / U_{j}^{2}$. Results for all three flow conditions are very similar and will be discussed in total.

Time-averaged axial velocity is plotted in figure 3. The LES agrees very well with the experimental data on both the centerline and lipline. The LES lies within the uncertainty bounds of the experimental data on the centerline, and is within or very close to the uncertainty bounds on the lipline. The RANS simulations have similar agreement on the lipline. But on the centerline, the location where the velocity begins to decay is predicted too far downstream for all cases. This indicates that the mixing layer between the jet and the freestream is spreading too slowly resulting in the inviscid core of the jet being too long. This behavior is typical of standard RANS models. ${ }^{16}$

Turbulent kinetic energy is plotted in figure 4. On the jet lipline, just downstream of the nozzle exit, the LES indicates very high values of $k / U_{j}^{2}$. This behavior has been reported previously ${ }^{17}$ and is due to the lack of a turbulent nozzle boundary layer in the LES. The spike in turbulent kinetic energy is due to the organized axisymmetric vortices that form in the mixing layer prior to transition. After transitioning, the $k / U_{j}^{2}$ recovers to the experimental values. On the centerline, the LES does a good job predicting the shape and levels of $k / U_{j}^{2}$. The LES over-predicts the peak values of $k / U_{j}^{2}$ for SP3 and SP27 on both the centerline and lipline. On the jet lipline the RANS simulations do not reproduce the humped nature of the curve. Instead of predicting an initial rise in $k / U_{j}^{2}$ over the first several diameters, the RANS shows a linear decrease. On the centerline the RANS simulations do not predict a gradual rise in turbulent kinetic energy and instead show a sharp increase at the end of the potential core.

Axial and radial turbulent intensities are plotted for the LES simulations in figures 5 and 6 . As expected, the axial intensities are higher than the radial intensities. As discussed above, the transitional region of the mixing layer can be seen in the sharp spikes in $u^{\prime} / U_{j}$ and $v^{\prime} / U_{j}$ just downstream of the nozzle lip. Overall the agreement with experiment is very good and the predictions lie within the uncertainty bounds of the data. In general, the radial intensities are slightly over-predicted. It is this over-prediction in $v^{\prime} / U_{j}$ that causes the over-prediction of $k / U_{j}^{2}$ in figure 4 .

Radial profiles of the LES derived turbulence intensities $u^{\prime} / U_{j} \& v^{\prime} / U_{j}$ are compared to the experiment in figure 7. Overall, very good agreement is seen between experiment and LES, and the data lies within the experimental uncertainty. The LES tends to over-predict the values of $v^{\prime} / U_{j}$ at all 3 set points, especially at $x / D_{j}=2$. For $u^{\prime} / U_{j}$ the LES shows excellent agreement at all set points and locations, with the exception of the downstream locations of SP 23. 


\section{Comparison to Temperature Data}

The LES and RANS solutions are compared to Mielke et al's Rayleigh scattering data and Locke \& Wernet's Raman spectroscopy data on the jet centerline and lipline in figures 8 and 9. Mean temperatures are plotted in the form of $\left|\left(\bar{T}-T_{\infty}\right)\right| / \Delta T_{j}$. The absolute value is used to make the cold jet data, where $T_{j}<T_{\infty}$, appear in the same form as the hot jet data. The mean temperature data behaves similarly to the mean velocity data (figure 3 ) with the exception that the centerline decay begins between 0.5 to 0.8 jet diameters earlier. For all cases, the RANS simulations predict a longer potential core than the LES and experimental data. It is interesting to note that for SP 3, the RANS and LES computations disagree on the lipline. On the lipline, the CFD methods agree on mean temperature for SPs $23 \& 27$ and on mean velocity for SPs $3,23 \& 27$. Experimental temperature measurements were not made for SP 3 due to the small difference between the jet and ambient temperatures. For SPs 23 and 27 there are differences between the Rayleigh and Raman techniques. On the centerline the Raman data is higher than the Rayleigh data for SP 23 and lower than the Rayleigh data for SP 27. On the lipline the Raman temperatures are higher for both set points with a larger difference at SP 23. On the centerline, the LES is in good agreement with the lower of the two experimental temperature measurements. On the lipline, both the LES and RANS predictions lie between the two experimental data sets.

Normalized turbulent temperature fluctuations, $T^{\prime} / \Delta T_{j}$ on the centerline and lipline are plotted in figure 9. The data appears similar to the fluctuating velocity data, but the rise on the centerline occurs earlier and the subsequent decay is more pronounced. The earlier rise in $T^{\prime} / \Delta T_{j}$ compared to $u^{\prime} / U_{j}$ and faster decay in $\left|\left(\bar{T}-T_{\infty}\right)\right| / \Delta T_{j}$ compared to $u / U_{j}$ indicates that the temperature mixing layer is spreading faster than the velocity mixing layer. This is expected for flows such as this, where the turbulent Prandtl number is less than one. Additional discussion of the turbulent Prandtl number is found in section F. As with the mean temperature data, there is some disparity between experimental measurement techniques. For SP 23, the Rayleigh obtained data is higher than the Raman data on both the centerline and lipline, with a significantly larger difference on the lipline. As noted earlier, there may be a spatial averaging effect in the Raman data due to the length of the laser pulse. This effect would reduce the values of $T^{\prime} / \Delta T_{j}$. For SP27, the Rayleigh data on the centerline is limited, but the values are also higher than the Raman data. However, on the lipline the Rayleigh data is lower than the Raman data. The LES predictions show the same form as the experimental data; the shape of the curves and the rise and decay of $T^{\prime} / \Delta T_{j}$ agree with the experiments. However, the levels from the LES predictions are consistently higher than the experiments. The lone exception is the lipline data for SP 23, where the LES agrees well with the Rayleigh scattering measurements.

Profiles of $\left|\left(\bar{T}-T_{\infty}\right)\right| / \Delta T_{j}$ and $T^{\prime} / \Delta T_{j}$ are shown in figures 10 and 11 , respectively. The Rayleigh scattering and Raman spectroscopy profiles were taken at different axial locations; only $x / D_{j}=2$ is common. For the mean temperature, the RANS solutions mix too slowly and exhibit the existence of the potential core at $x / D_{j}=6$ where the data and LES show that it has decayed. In general there is good agreement between LES and experiment. For the temperature fluctuations, the LES and experiments exhibit the same trends. The LES tends to predict higher levels than the experiment, although there are some exceptions at a few locations where Rayleigh scattering data was taken.

\section{Evaluation of the Turbulent Heat Flux}

The LES computation of the turbulent heat flux vector is examined next. The comparisons between LES and experiment discussed above show that the LES does a very good job predicting mean and fluctuating velocities. They also show that while the LES tends to over-predict the levels of the temperature fluctuations, the shape of the curves and general behavior of the temperature field is accurately reproduced. This should establish that the LES is correctly reproducing the behavior of the jets and should lend confidence that the combined quantity $\overline{\rho u^{\prime} T^{\prime}}$ is reasonably predicted by the LES.

Profiles of the normalized axial, $q_{x}^{T} /\left(\rho_{j} U_{j} \Delta T_{j}\right)$, and radial, $q_{r}^{T} /\left(\rho_{j} U_{j} \Delta T_{j}\right)$, components of the turbulent heat flux vector for the 6 downstream locations are plotted in figure 12 for both the LES and RANS simulations. For SP 3 the jet temperature is lower than the ambient temperature and therefore the sign of the heat flux is opposite to SP $23 \& 27$, where the jet temperature is greater than the ambient. There is very good agreement between LES and RANS for the radial component of the heat flux for all three set points. Both the magnitudes and shapes of the profiles match well and the differences at a given axial location can be largely attributed to the differences in the spreading rate of the mixing layer and difference in the length 
of the potential core. There is no agreement between LES and RANS for the axial component of the heat flux. The LES results show that the axial component is similar in shape and larger in magnitude than the radial component. The RANS values are an order-of-magnitude smaller, and have both positive and negative values.

There is prior experimental evidence to suggest that the LES derived heat flux is the more correct result. Pope cites two different studies in his discussion of scalar transport in free shear flows. ${ }^{37}$ Both studies used a combination of hot wire anemometry and cold wire thermometry to obtain their data. 1) Fabris ${ }^{2}$ measured the heat flux vector in the wake of a heated cylinder. His results indicated that the magnitudes of the axial and transverse components of the vector have similar magnitudes. 2) Tavoularis \& Corrsin studied a turbulent shear flow with an imposed mean temperature gradient. ${ }^{3}$ They found that the axial component was larger than the transverse component.

Pope, in his book Turbulent Flows ${ }^{37}$ discusses the consequences of the gradient diffusion based heat flux model used in RANS. As seen in equation 9, the heat flux vector must be aligned with the mean temperature gradient. For a round jet the mean temperature gradient is nearly normal to the jet centerline. As a consequence the axial component of the RANS heat flux vector must be small. The large value of $q_{x} /\left(\rho_{j} U_{j} \Delta T_{j}\right)$ in the LES indicates that the heat flux vector is far from aligned with the temperature gradient. Analysis of the LES flow fields indicate that the direction of the mean temperature gradient varies from 85-95 degrees from the jet axis. The direction of the heat flux vector varies from 29-37 degrees from the jet axis. The angle between the two vectors is approximately 57 degrees. In Tavoularis \& Corrsin's experiment, ${ }^{3}$ the angle between the temperature gradient and heat flux vector was 63 degrees. ${ }^{37}$ It is apparent that the gradient diffusion model is not appropriate for shear flows.

The Reynolds stress and turbulent heat flux contours are compared in figure 13. The bulge in the shear layer and high contour levels, just downstream of the nozzle exit, are a result of the two-dimensional structures due to the transitional state of the mixing layer. The contours all peak in the center of the mixing layer, just downstream of the end of the potential core. The axial normal stress (figure 13a) and axial turbulent heat flux (figure 13c) display very similar structure. Downstream of the potential core $\left(x / D_{j} \geq 6\right.$ ), as $r / D_{j}$ approaches zero the contour levels are reduced but do not approach zero. The shear stress (figure 13b) and radial turbulent heat flux (figure 13d) also display very similar structure. Downstream of the potential core $\left(x / D_{j} \geq 6\right)$, as $r / D_{j}$ approaches zero, the contour levels also approach zero, and beyond $x / D_{j}=10$ the contours lift off the centerline. Set points 3 and 23 show the same behavior. This indicates that the turbulent transport of temperature behaves similarly to the turbulent transport of axial momentum. This agrees with the conclusion of Tavoularis \& Corrsin. ${ }^{3}$ This similarity may provide insight for new heat flux models.

\section{E. Effect of the Turbulent Heat Flux on the Energy Equation}

Next we examine the effect of the disparity in the RANS and LES axial turbulent heat flux terms on the energy equation. It is the gradient of the turbulent heat flux vector, $\partial q_{i}^{T} / \partial x_{i}$, that appears in the energy equation (equation 4). The axial and radial gradient terms are $\partial q_{x}^{T} / \partial x$ and $\partial q_{r}^{T} / \partial r$. To quantify the contribution of the axial term, its magnitude was normalized with respect to the total contribution of the heat flux gradient terms.

$$
\left\|\frac{\partial q_{x}^{T}}{\partial x}\right\|=\frac{\left|\frac{\partial q_{x}^{T}}{\partial x}\right|}{\sqrt{\left(\frac{\partial q_{x}^{T}}{\partial x}\right)^{2}+\left(\frac{\partial q_{r}^{T}}{\partial r}\right)^{2}}}
$$

Outside of the shear layer, the heat flux gradient terms have small, but nonzero, values. These small terms can produce large values of the normalized magnitude of the axial gradient of the axial heat flux term, which obfuscate the data. In order to focus on the data in the shear layer, the value of $\left\|\partial q_{x}^{T} / \partial x\right\|$ was set to zero as $q_{x}^{T}$ approached zero. The data in the following plots exhibit noise. The time averaged LES data contains noise, which is not discernible in the previous plots. The derivative and division operations in equation 10 exacerbate this noise.

For RANS, $q_{x}^{T}$ is essentially zero and has no discernible effect on the energy equation (see figure 12). For LES, profiles of $\left\|\partial q_{x}^{T} / \partial x\right\|$ are plotted in figure 14. The plots show for all cases that near the edges of the shear layer the axial gradient term accounts for approximately 10 to 20 percent of the total turbulent heat flux contribution. Near the center of the mixing layer the axial gradient term dominates. This is due to the fact that $\partial q_{r}^{T} / \partial r$ goes to zero in the center of the mixing layer. The data show that by using the standard gradient diffusion model in RANS, a small but significant contribution to the energy equation is neglected. 


\section{F. Evaluation of the Turbulent Prandtl Number}

A key component in the turbulent heat flux model (equation 9) is the turbulent Prandtl number. For most RANS calculations, $P r_{t}$ is fixed at a constant value. This value is dependent on the type of flow and can vary within the flow. For a boundary layer, $P r_{t}$ varies from 0.9 near the wall to 0.7 near the boundary layer edge. For many free shear flows, $P r_{t}$ is approximately 0.5 . For round jets $P r_{t}$ is typically assumed to be $0.7,^{38,39}$ however other values are regularly reported. The LES data was used to evaluate the turbulent Prandtl number in the flow fields considered here. The momentum and temperature diffusivities were computed from the radial fluxes and the mean radial gradients. The mean gradients were assumed to be strictly in the radial direction.

$$
\epsilon_{m}=-\frac{\overline{\rho u^{\prime} v^{\prime}}}{\bar{\rho} \frac{\partial \bar{u}}{\partial r}}
$$

$$
\epsilon_{T}=-\frac{\overline{\rho v^{\prime} T^{\prime}}}{\bar{\rho} \frac{\partial \bar{T}}{\partial r}}
$$

and the turbulent Prandtl number was computed from their ratio.

$$
\operatorname{Pr}_{t}=\frac{\epsilon_{m}}{\epsilon_{T}}
$$

The calculation of the turbulent Prandtl number suffers from the same problems as equation 10; spurious values outside of the shear layer and noise. For clarity, $P r_{t}$ was set to zero as the radial temperature gradient approaches zero.

Radial profiles of the LES derived $P r_{t}$ are shown in figure 15. All three cases exhibit very similar characteristics. Near the edges of the shear layer, the values have a tendency to attain extreme values. For the purpose of clarity these values were limited to $-0.5 \leq P r_{t} \geq 1.5$ in the plots. For the majority of the shear layer $\operatorname{Pr}_{t}$ is close to the accepted value for round jets of 0.7. The profiles near the nozzle exit, $x / D_{j}=2 \& 4$, exhibit some variation, in the form of a double hump. The downstream profiles are relatively constant. The data appears to indicate that the assumption of a constant turbulent Prandtl number of 0.7 is reasonable.

\section{Summary and Conclusions}

A computational investigation of the turbulent temperature fluctuations and turbulent heat flux in hot jets was carried out. Reynolds-averaged Navier-Stokes (RANS) computations using the Wind-US code, and large-eddy simulations (LES) using a high-order finite-difference code, WRLES, were carried out for jets emanating from a 2-inch round nozzle. Three flow conditions were examined: a low-speed cold jet, a lowspeed hot jet, and a high-speed hot jet. The computational results were compared to experimental data from three sources: 1) Mean velocity and turbulence intensities measurements using particle image velocimetry, 2) Mean and fluctuating temperature measurements using Rayleigh scattering, and 3) Mean and fluctuating temperature measurements from Raman spectroscopy. The RANS simulations did not accurately predict the jet flowfield. As is typical, the RANS solutions under-predict the turbulent mixing rate, resulting in a potential core that is too long. The LES results were in very good agreement for mean velocities and turbulence intensities. The LES also accurately predicted the correct mean and fluctuating temperature behavior. However, the LES over-predicted the levels of temperature fluctuation. It was also found that there are significant differences in the levels of the two experimental temperature datasets. The comparison of the LES results to experiment lends confidence that the turbulent heat flux is reasonably well predicted by the LES.

Turbulent heat flux was computed for the RANS and LES computations and compared. Both simulations produced very similar results for the radial component of the heat flux vector. However, the axial component of the turbulent heat flux vector, obtained from the LES, differed significantly from the RANS result in both profile shape and magnitude. The RANS based quantity was nearly zero, whereas the LES based quantity was of similar profile shape and larger in magnitude than the radial heat flux. Previous experimental studies lend confidence to the LES result. It was noted that the gradient diffusion type model used in the RANS analyses can not produce a significant axial heat flux component because it is based on the mean axial temperature gradient, which is nearly zero for a round jet. The effect of the axial component of the heat flux vector on the energy equation was quantified and compared to the effect of the radial component. Because the gradient of the heat flux vector is used in the energy equation the overall effect of the error in the RANS 
model is tempered. Results show the axial component of the heat flux vector nominally comprises 10 to 20 percent of the total contribution by the turbulent heat flux. Finally, the LES data was used to compute the turbulent Prandtl number. Results showed that the turbulent Prandtl number varied between 0.65 and 0.75 for the majority of the jet's mixing layer, verifying that the standard value of 0.7 for round jets is reasonable.

\section{Acknowledgements}

I would like to thank Dr. Dennis Yoder, Dr. Nicholas Georgiadis, Dr. Mark Wernet and Dr. Amy (Mielke) Fagan for their advice and assistance with this work. This research was sponsored by the National Aeronautics and Space Administration's Transformational Tools and Technologies Project. All the computer simulations were performed on the Pleiades Supercomputer, part of NASA's High-End Computing Capability.

\section{References}

\footnotetext{
${ }^{1}$ Slotnick, J., Khodadoust, A., Alonso, J., Darmofal, D., Gropp, W., Lurie, E., and Mavriplis, D., "CFD Vision 2030 Study: A Path to Revolutionary Computational Aerosciences," NASA CR 2014-218178, 2014.

${ }^{2}$ Fabris, G., "Conditional Sampling Study of the Turbulent Wake of a Cylinder. Part 1," Journal of Fluid Mechanics, Vol. 94, 1979, pp. 673-709.

${ }^{3}$ Tavoularis, S. and Corrsin, S., "Experiments in Nearly Homogenous Turbulent Shear Flow with a Uniform Mean Temperature Gradient. Part 1," Journal of Fluid Mechanics, Vol. 104, 1981, pp. 311-347.

${ }^{4}$ Sohn, K., Zaman, K., and Reshotko, E., "Turbulent Heat Flux Measurements in a Transitional Boundary Layer," NASA TM 105623, 1992.

${ }^{5}$ Nagata, K., Sakai, Y., and Komori, S., "Effects of Small-Scale Freestream Turbulence on Turbulent Boundary Layers With and Without Thermal Convection," Physics of Fluids, Vol. 23, No. 065111, 2011, pp. 1-14.

${ }^{6}$ Lomas, C., Fundamentals of Hot Wire Anemometry, Cambridge University Press, 1985, p. 178.

${ }^{7}$ Horstman, C. and Rose, W., "Hot-Wire Anemometry in Transonic Flows," NASA TM X-62,495, 1975.

${ }^{8}$ Lau, J. C., Morris, P. J., and Fisher, M. J., "Measurements in Subsonic and Supersonic Free Jets Using a Laser Velocimeter," Journal of Fluid Mechanics, Vol. 93, 1979, pp. 1-27, part 1.

${ }^{9}$ Bridges, J. E. and Wernet, M. P., "Measurements of the Aeroacoustic Sound Source in Hot Jets," AIAA Paper 2003-3130, 2003.

${ }^{10}$ Seasholtz, R., Buggele, A., and Reeder, M., Instantaneous Measurements in a Supersonic Wind Tunnel Using Spectrally Resolved Rayleigh Scattering, Society of Photo- Optical Instrumentation Engineers, Bellingham, Washington, 1995.

${ }^{11}$ Mielke, A. and Elam, K., Dynamic Measurement of Temperature, Velocity, and Density in Hot Jets Using Rayleigh Scattering, Proceedings of the International Symposium on Optical Science, Engineering and Instrumentation, Lisbon, Portugal, 2008.

${ }^{12}$ Cutler, A., Danehy, P., Springer, R., O’Byrne, S., D.P. Capriotti, D. P., and Deloach, R., "Coherent Anti-Stokes Raman Spectroscopic Thermometry in a Supersonic Combustor," AIAA Journal, Vol. 41, No. 12, 2003, pp. 2451-2459.

${ }^{13}$ Woodmansee, M., Iyer, V., Dutton, J., and Lucht, R., "Nonintrusive Pressure and Temperature Measurements in an Underexpanded Sonic Jet Flowfield," AIAA Journal, Vol. 42, No. 6, 2004, pp. 1170-1180.

${ }^{14}$ Kuehner, J., Tessier, F. A., Kisoma, A., Flittner, J., and McErlean, M., "Measurements of Mean and Fluctuating Temperature in an Underexpanded Jet Using Electrostrictive Laser-Induced Gratings," Experiments in Fluids, Vol. 48, No. 3, 2010, pp. 421-430.

${ }^{15}$ Dembowski, M. A. and Georgiadis, N. J., "An Evaluation of Parameters Influencing Jet Mixing Using the WIND NavierStokes Code," NASA TM 2002-211727, 2002.

${ }^{16}$ Georgiadis, N. J. and DeBonis, J. R., "Navier-Stokes Analysis Methods for Turbulent Jet Flows with Application to Aircraft Exhaust Nozzles," Progress in the Aerospace Sciences, Vol. 42, 2006, pp. 377-418.

${ }^{17}$ DeBonis, J. R., "A High-Resolution Capability for Large-Eddy Simulation of Jet Flows," AIAA Paper 2010-5023, 2010.

${ }^{18}$ Bogey, C. and Marsden, O., "Identification of the Effects of the Nozzle-Exit Boundary-Layer Thickness and its Corresponding Reynolds Number in Initially Highly Disturbed Subsonic Jets," Physics of Fluids, Vol. 25, No. 055106, 2013.

${ }^{19}$ Ingraham, D. and Bridges, J., "Validating a Monotonically-Integrated Large Eddy Simulation Code for Subsonic Jet Acoustics," AIAA Paper 2017-0456, 2017.

${ }^{20}$ Bridges, J. and Wernet, M. P., "The NASA Subsonic Jet Particle Image Velocimetry (PIV) Dataset," NASA TM 2011216807, 2011.

${ }^{21}$ Mielke, A., Elam, K., and Sung, C.-J., "Multiproperty Measurements at High Sampling Rates Using Rayleigh Scattering," AIAA Journal, Vol. 47, No. 4, 2009, pp. 850-862.

${ }^{22}$ Locke, R., Wernet, M., and Anderson, R., "Rotational Raman-Based Temperature Measurements in a High-Velocity Turbulent Jet," NASA TM 2017-219504, 2017.

${ }^{23}$ Menter, F. R., "Two-Equation Eddy-Viscosity Turbulence Models for Engineering Applications," AIAA Journal, Vol. 32, No. 8, 1994, pp. 1598-1605.

${ }^{24}$ Spalart, P. R. and Allmaras, S. R., "A One-Equation Turbulence Model for Aerodynamic Flows," La Recherche Aerospatiale, , No. 1, 1994, pp. 5-21.

${ }^{25}$ Jones, W. P. and Launder, B. E., "The Prediction of Laminarization with a Two-Equation Model of Turbulence," International Journal of Heat and Mass Transfer, Vol. 15, 1972, pp. 301-314.
} 
${ }^{26}$ Yoder, D., "Comparison of Turbulent Thermal Diffusivity and Scalar Variance Models," AIAA Paper 2016-1561, 2016.

${ }^{27}$ Tanna, H., Dean, P., and Burrin, R., "The Generation and Radiation of Supersonic Jet Noise, Volume III, Turbulent Mixing Noise Data," Air Force Aero-Propulsion Laboratory Technical Report AFAPL-TR-76-65, 1976.

${ }^{28}$ DeBonis, J. R. and Scott, J. N., "A Large-Eddy Simulation of a Turbulent Compressible Round Jet," AIAA Journal, Vol. 40, No. 5, 2002, pp. 1346-1354.

${ }^{29}$ DeBonis, J. R., "A Large-Eddy Simulation of a High Reynolds Number Mach 0.9 Jet," AIAA Paper 2004-3025, 2004.

${ }^{30}$ DeBonis, J. R., "An Examination of the Spatial Resolution Requirements for LES of a Compressible Jet," Quality and Reliability of Large-Eddy Simulation II, edited by M. V. Salvetti, B. Geurts, H. Meyers, and P. Sagaut, Springer, 2010 , pp. 329-338.

${ }^{31}$ Bogey, C. and Bailly, C., "A Family of Low Dispersive and Low Dissipative Explicit Schemes for Flow and Noise Computations," Journal of Computational Physics, Vol. 194, 2004, pp. 194-214.

${ }^{32}$ Carpenter, M. H. and Kennedy, C. A., "Fourth-Order 2N-Storage Runge-Kutta Schemes," NASA TM 109112, 1994.

${ }^{33}$ Pointwise, Inc., http://www.pointwise.com.

${ }^{34}$ Kennedy, C. A. and Carpenter, M. H., "Comparison of Several Numerical Methods for Simulation of Compressible Shear Layers," NASA TP 3484, 1997.

${ }^{35}$ The NPARC Alliance, http://www.grc.nasa.gov/www/winddocs/.

${ }^{36}$ Menter, F. R., "Improved Two-Equation k-omega Turbulence Models for Aerodynamic Flows," NASA TM 1992-103975, 1992.

${ }^{37}$ Pope, S. B., Turbulent Flows, Cambridge University Press, 2000, pp. 161-167.

${ }^{38}$ Reynolds, A., Turbulent Flows in Engineering, John Wiley \& Sons, 1974, pp. 249-254.

${ }^{39}$ Hinze, J., Turbulence, McGraw-Hill, 1975, pp. 534-545.

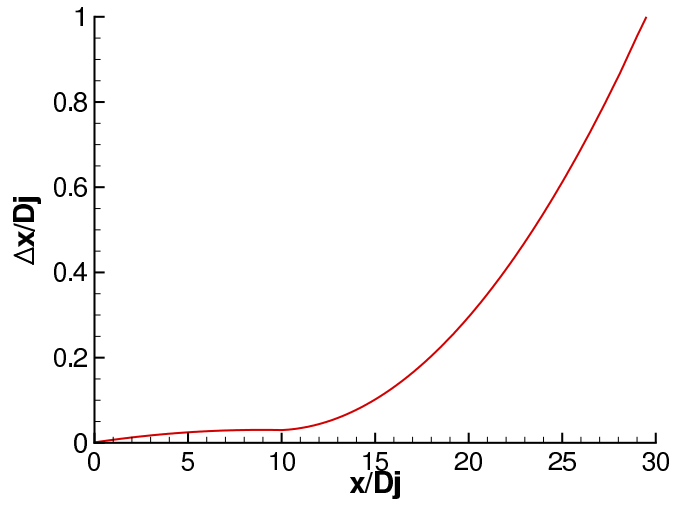

(a) Axial grid spacing

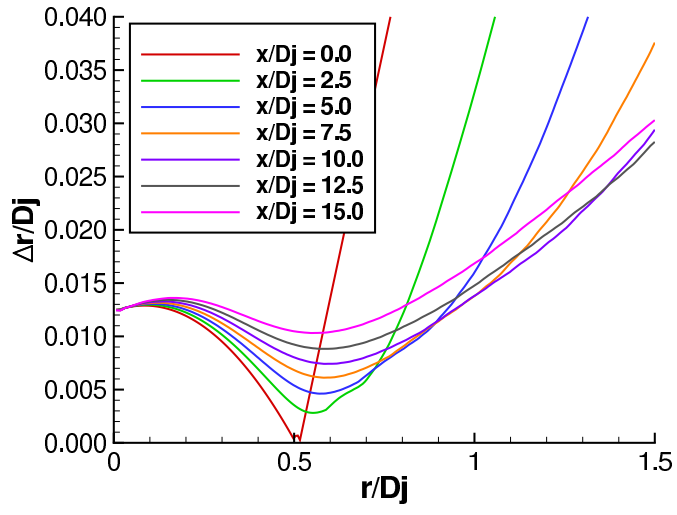

(b) Radial grid spacings downstream of the nozzle exit

Figure 1: Axial and radial grid spacing

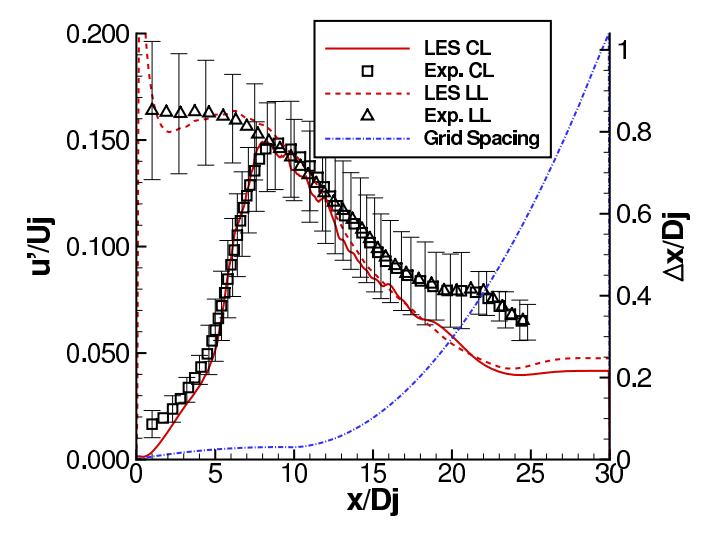

Figure 2: Effect of axial grid spacing on solution accuracy 


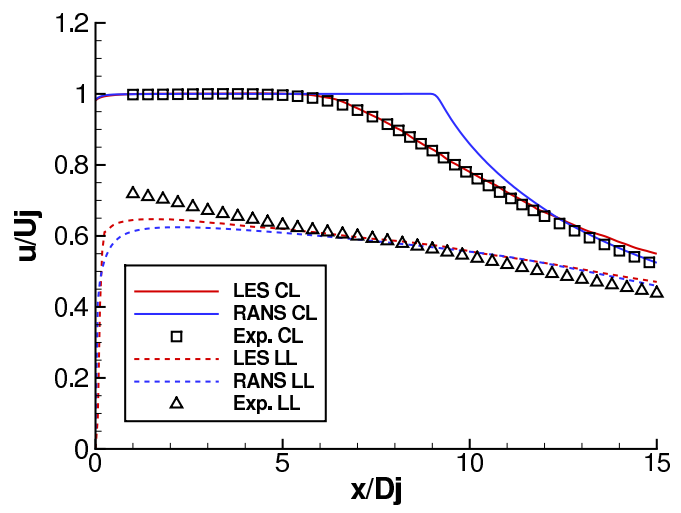

(a) SP 3

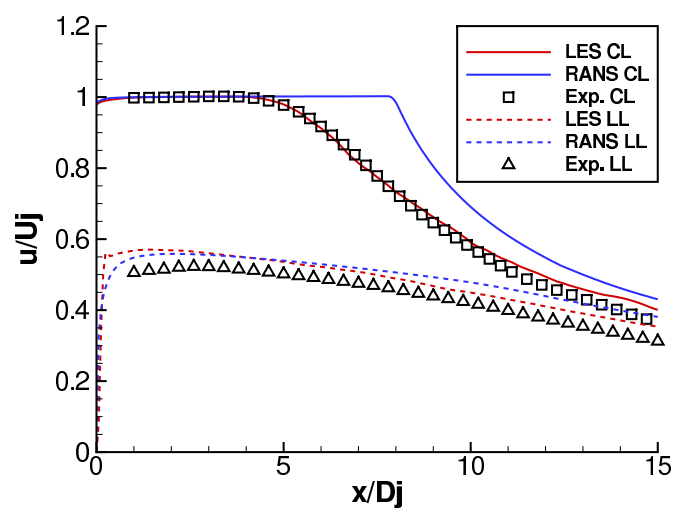

(b) SP 23

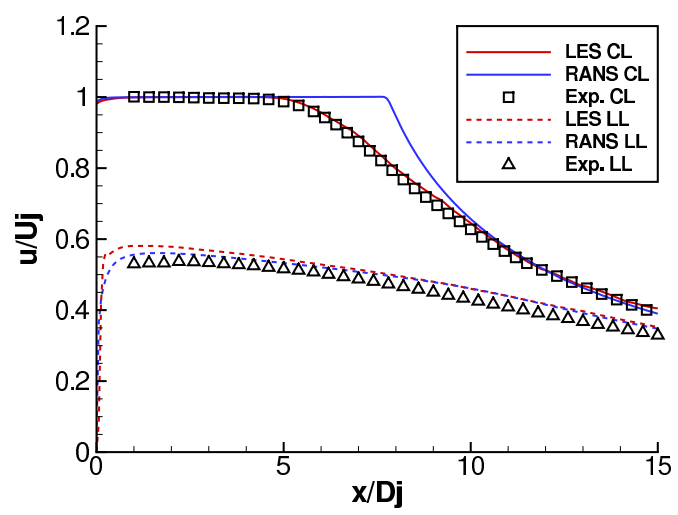

(c) SP 27

Figure 3: Time-averaged axial velocity, $\bar{u} / U_{j}$, on centerline and lipline 


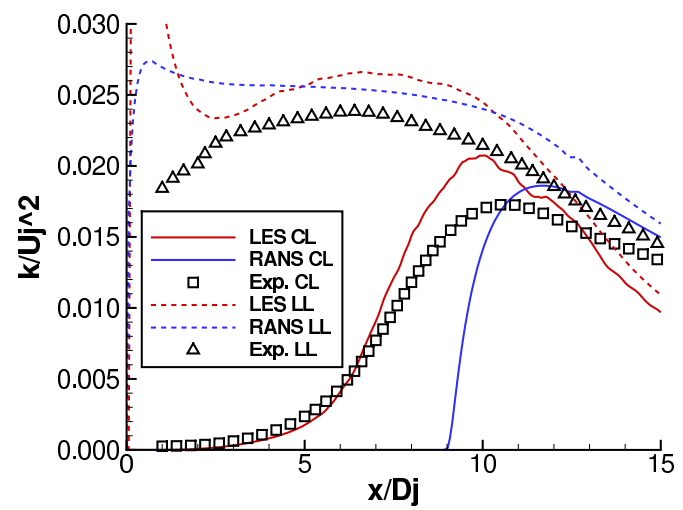

(a) SP 3

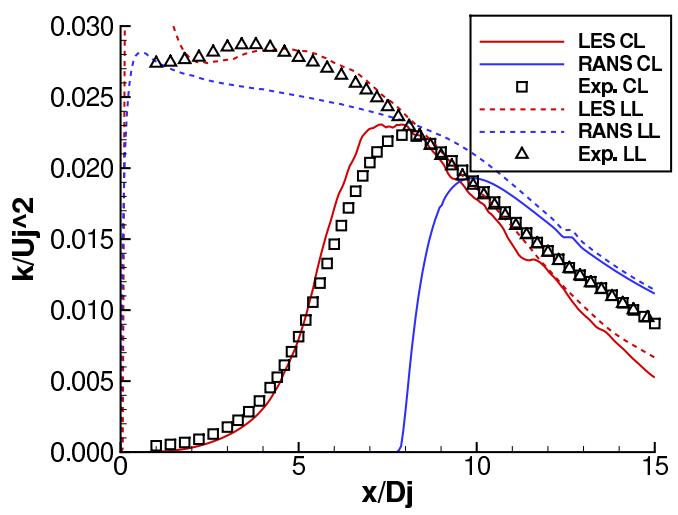

(b) SP 23

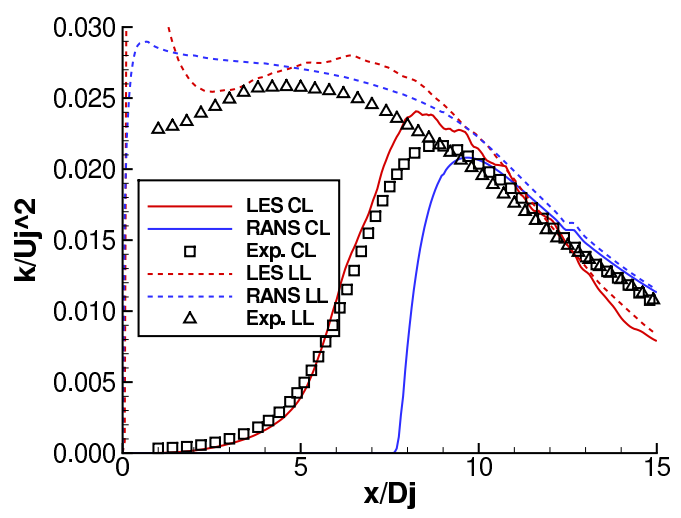

(c) SP 27

Figure 4: Turbulent kinetic energy, $k / U_{j}^{2}$, on centerline and lipline 


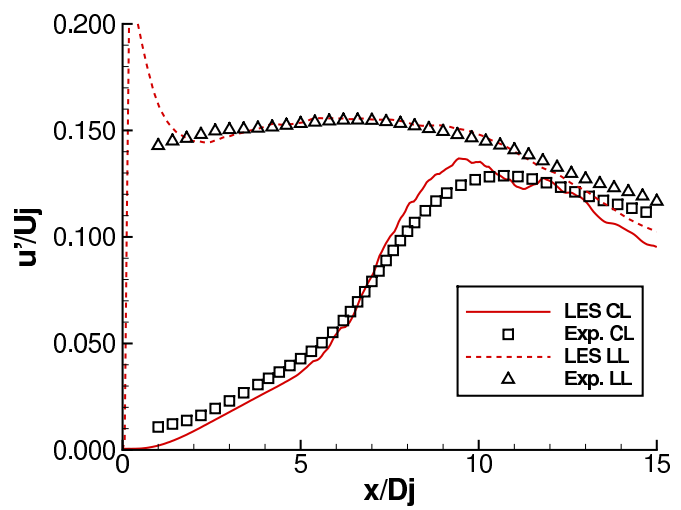

(a) SP 3

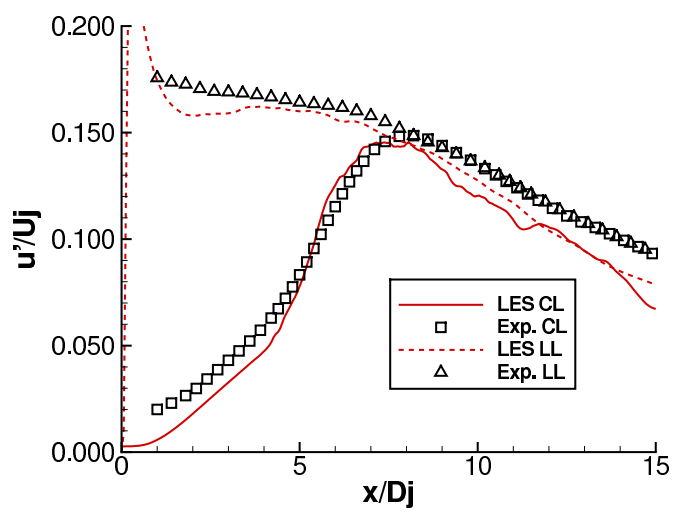

(b) SP 23

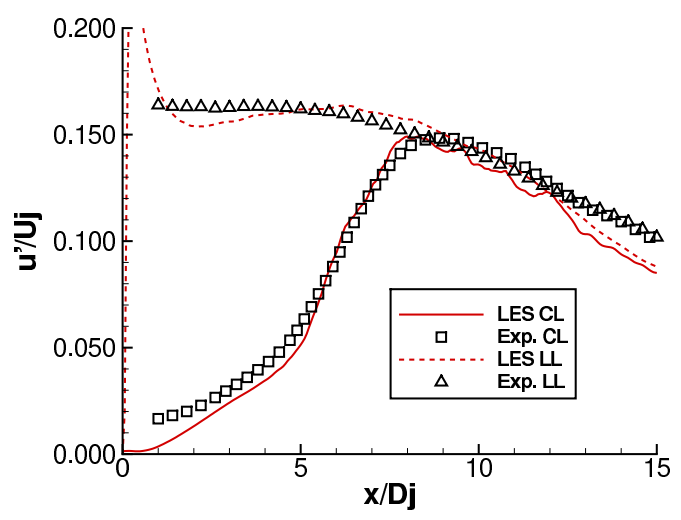

(c) SP 27

Figure 5: Axial turbulence intensity, $u^{\prime} / U_{j}$, on centerline and lipline 


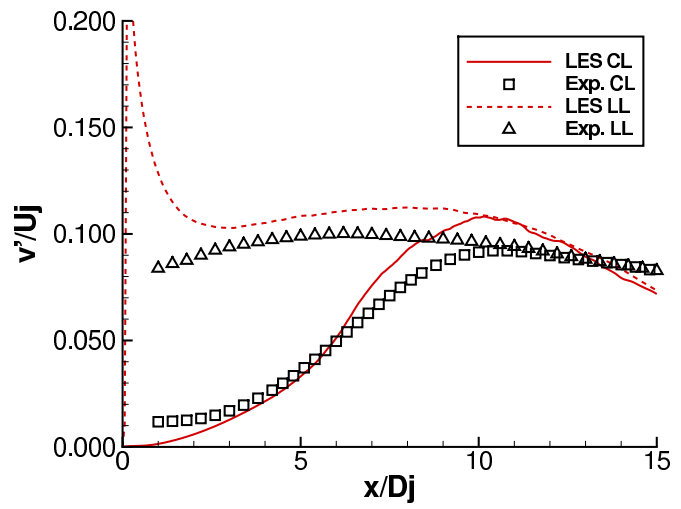

(a) SP 3

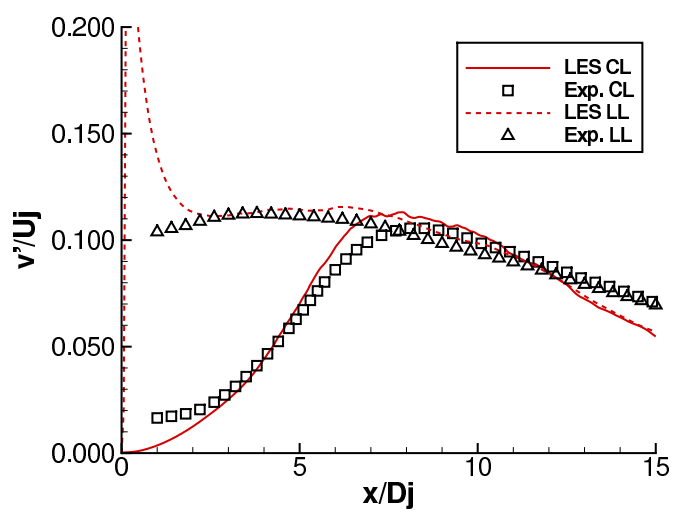

(b) SP 23

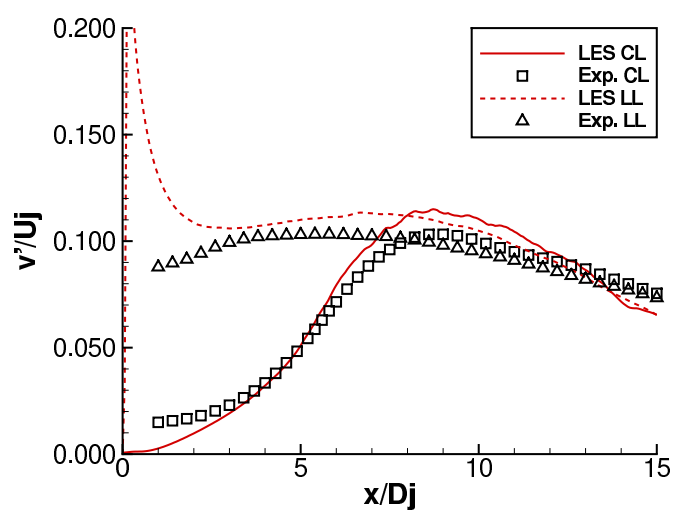

(c) SP 27

Figure 6: Radial turbulence intensity, $v^{\prime} / U_{j}$, on centerline and lipline 


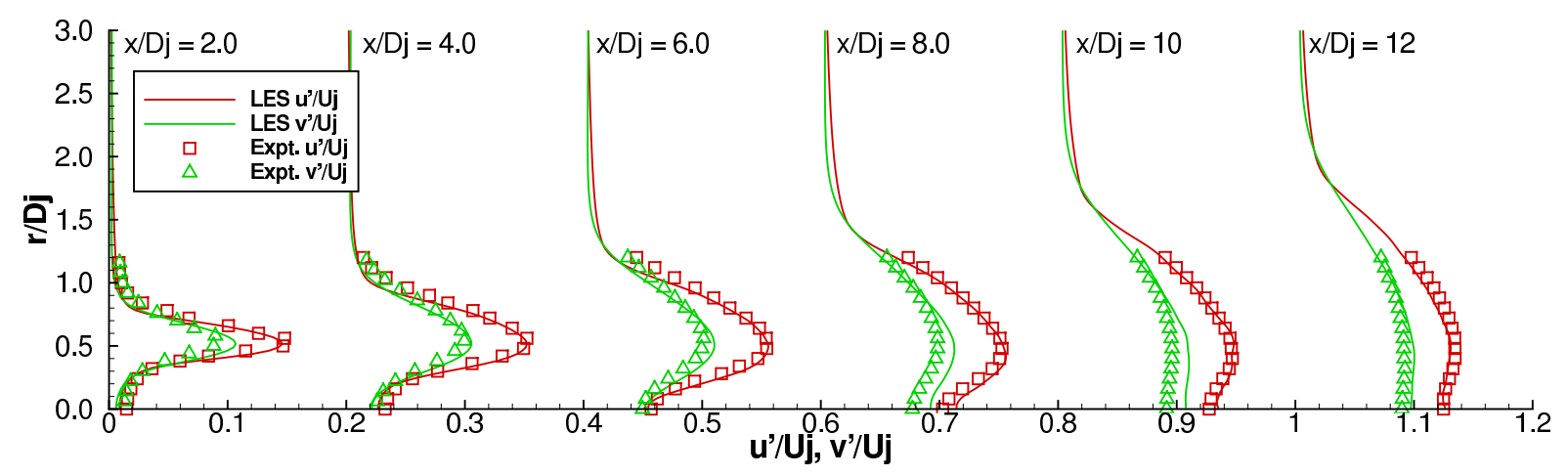

(a) SP 3

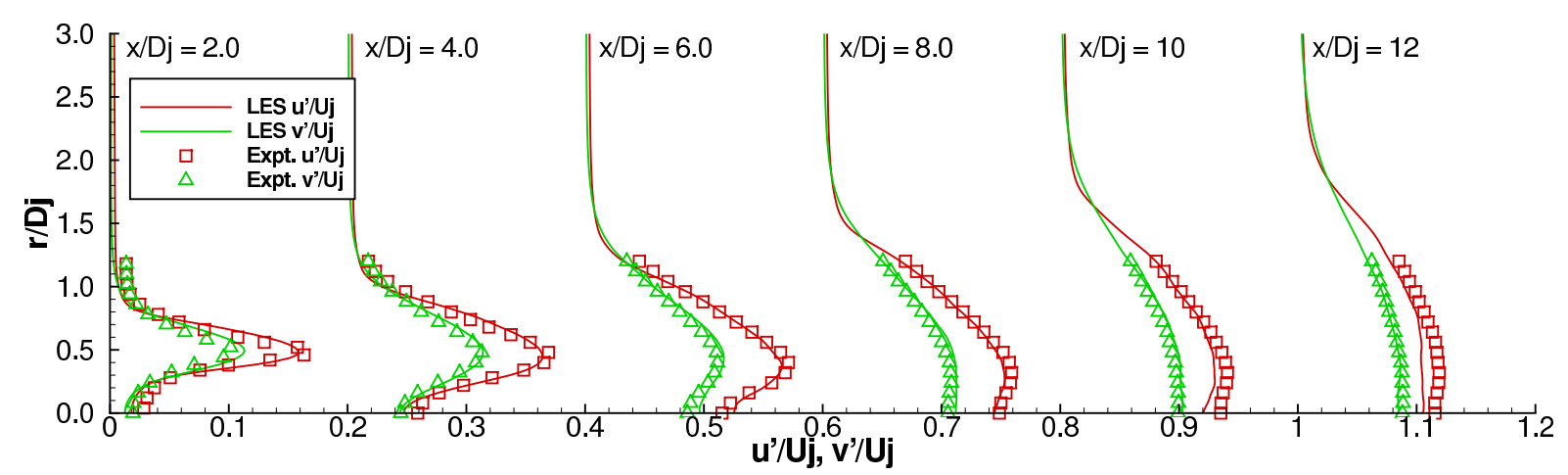

(b) SP 23

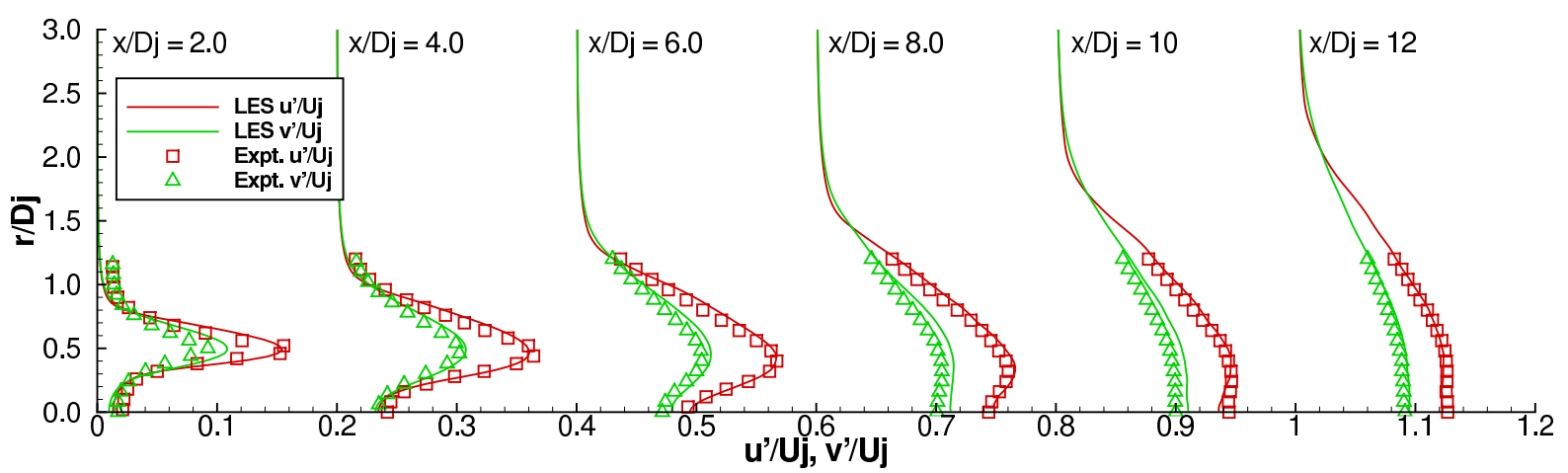

(c) SP 27

Figure 7: Radial profiles of the fluctuating velocities, $u^{\prime} / U j \& v^{\prime} / U j$. Each profile shifted 0.2 units. 


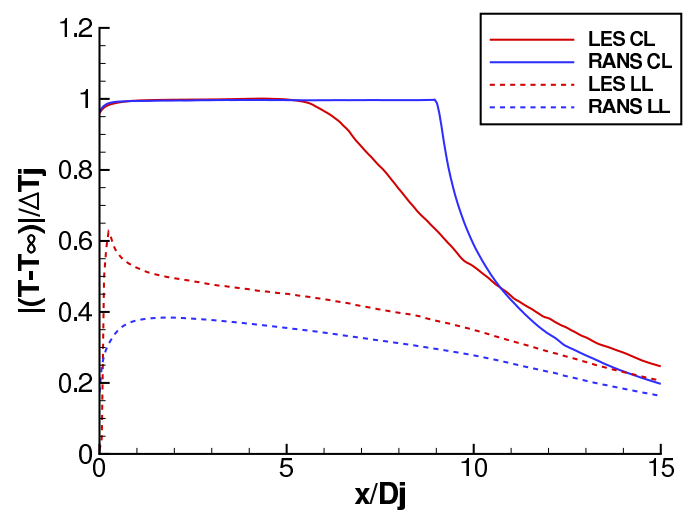

(a) SP 3

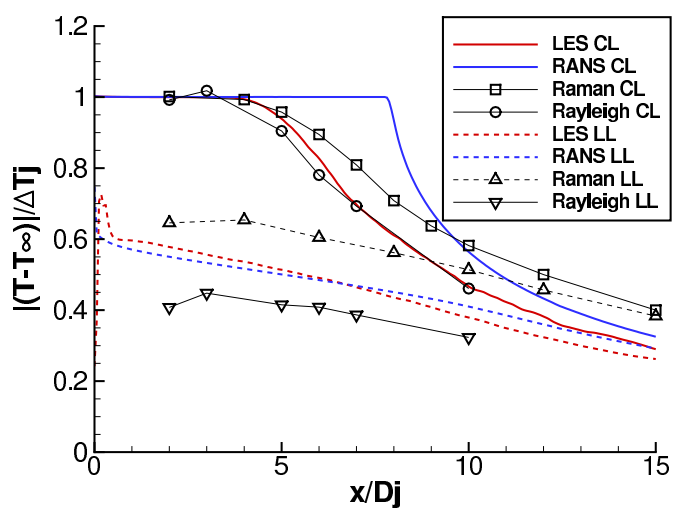

(b) SP 23

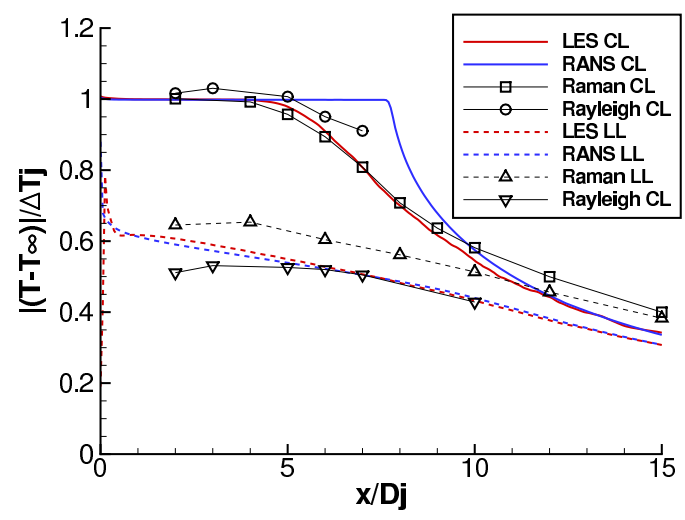

(c) SP 27

Figure 8: Time averaged temperature, $\left|\left(\bar{T}-T_{\infty}\right)\right| / \Delta T_{j}$, on centerline and lipline. 


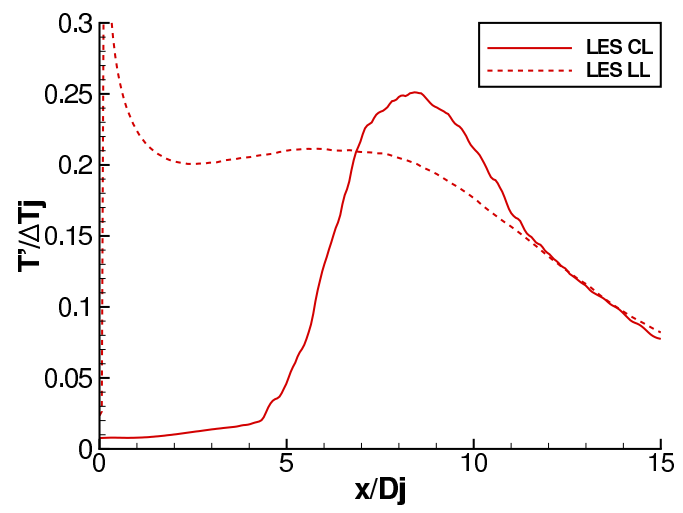

(a) SP 3

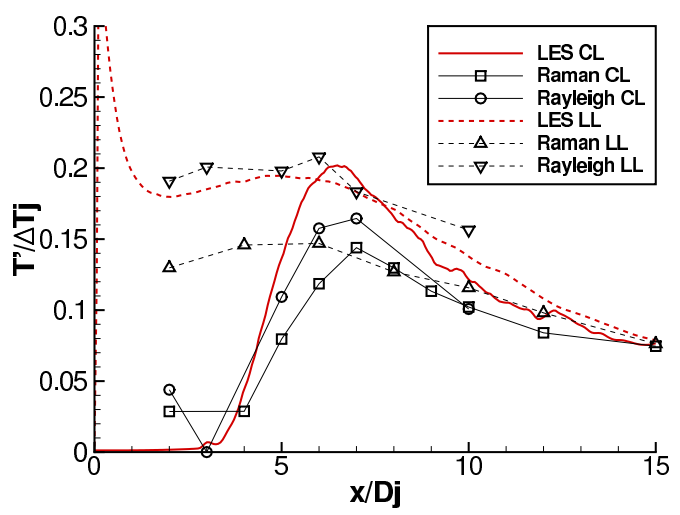

(b) SP 23

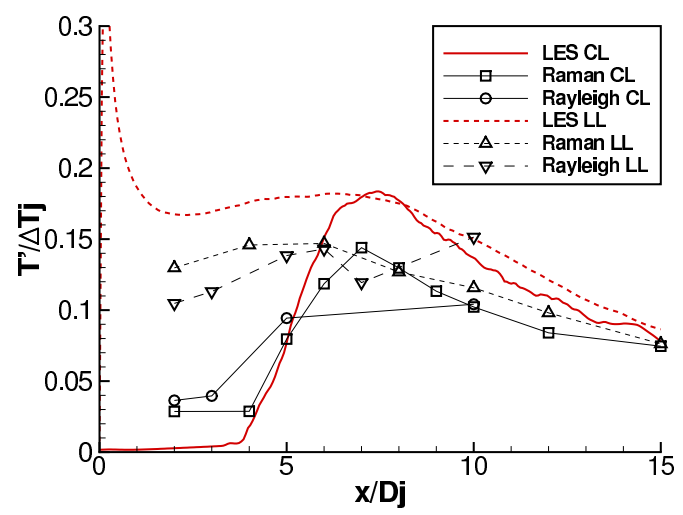

(c) SP 27

Figure 9: Fluctuatiing temperature, $T^{\prime} / \Delta T_{j}$, on centerline and lipline 


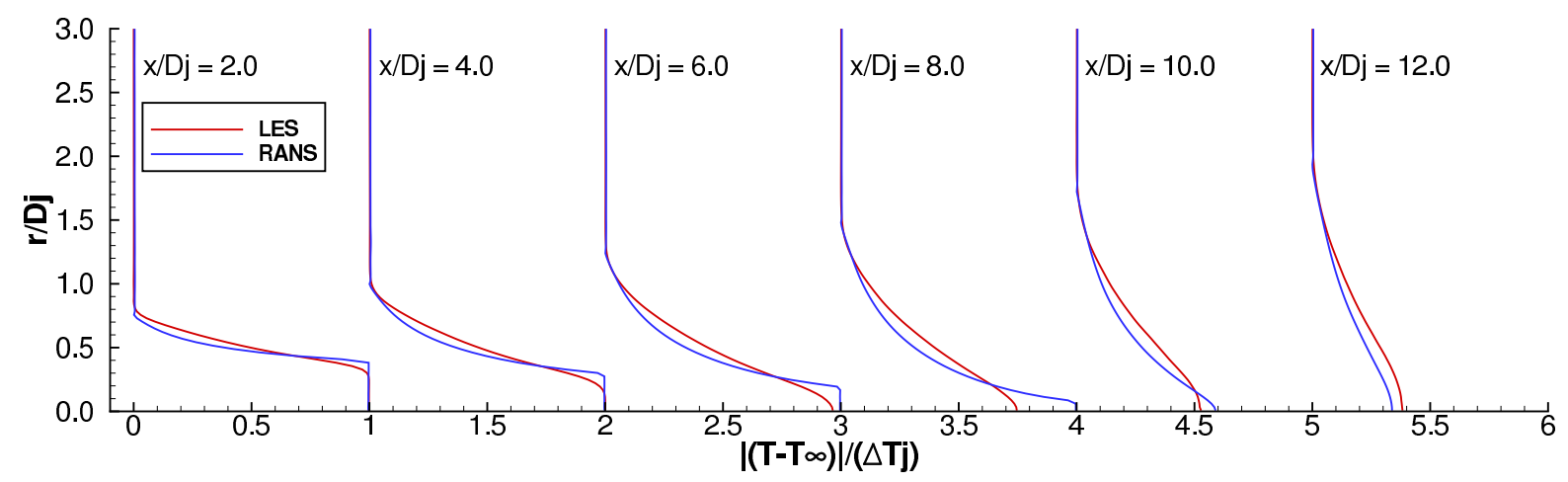

(a) SP 3

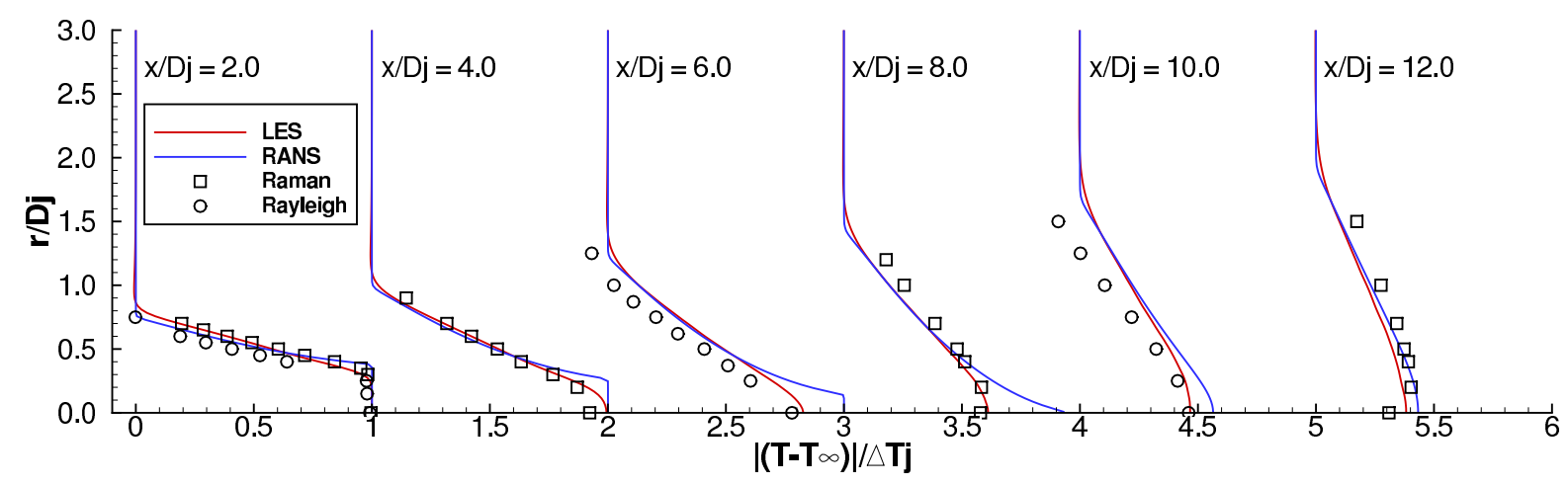

(b) SP 23

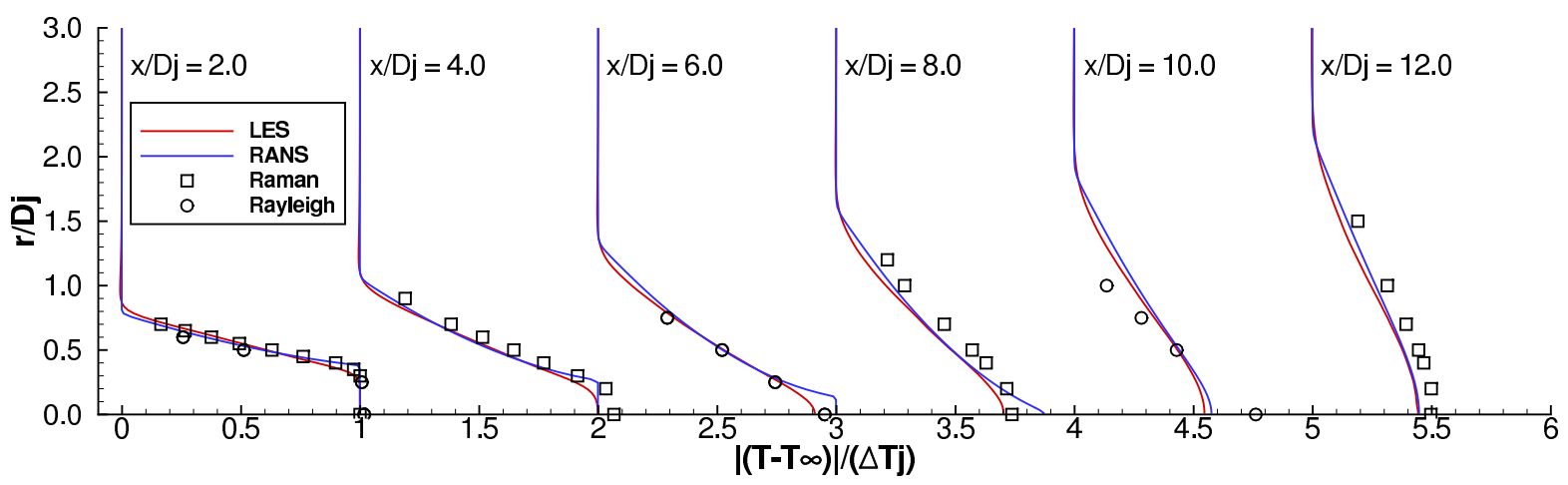

(c) SP 27

Figure 10: Radial profiles of the time averaged temperature, $\left|\left(\bar{T}-T_{\infty}\right)\right| / \Delta T_{j}$. Each profile shifted 1 unit. 


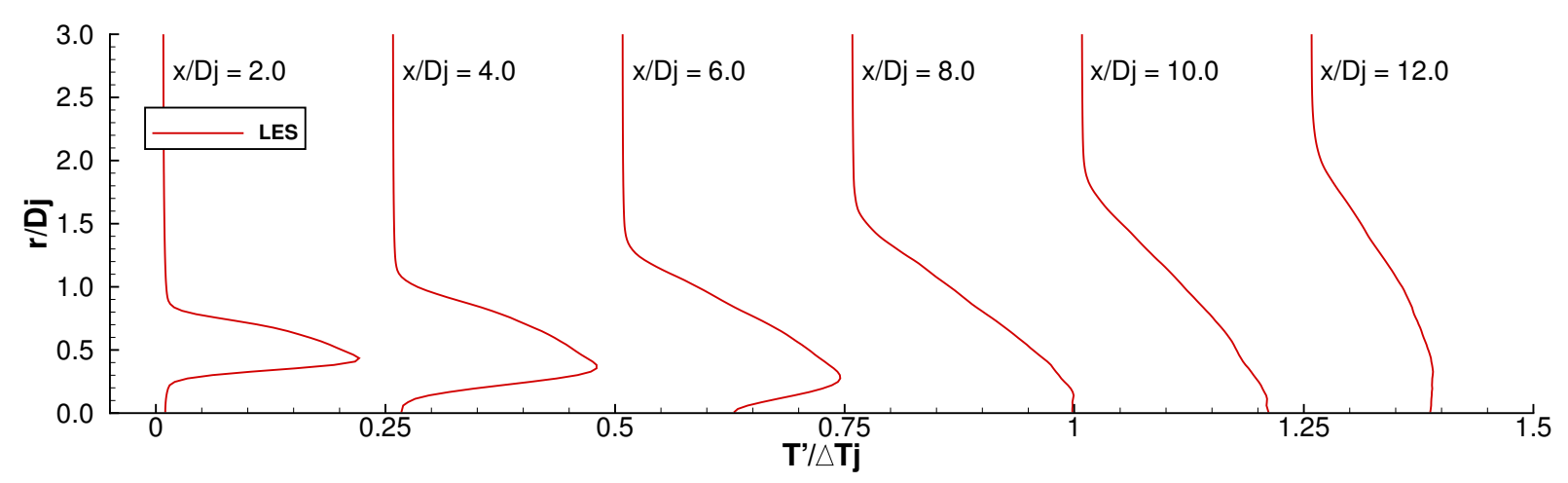

(a) SP 3

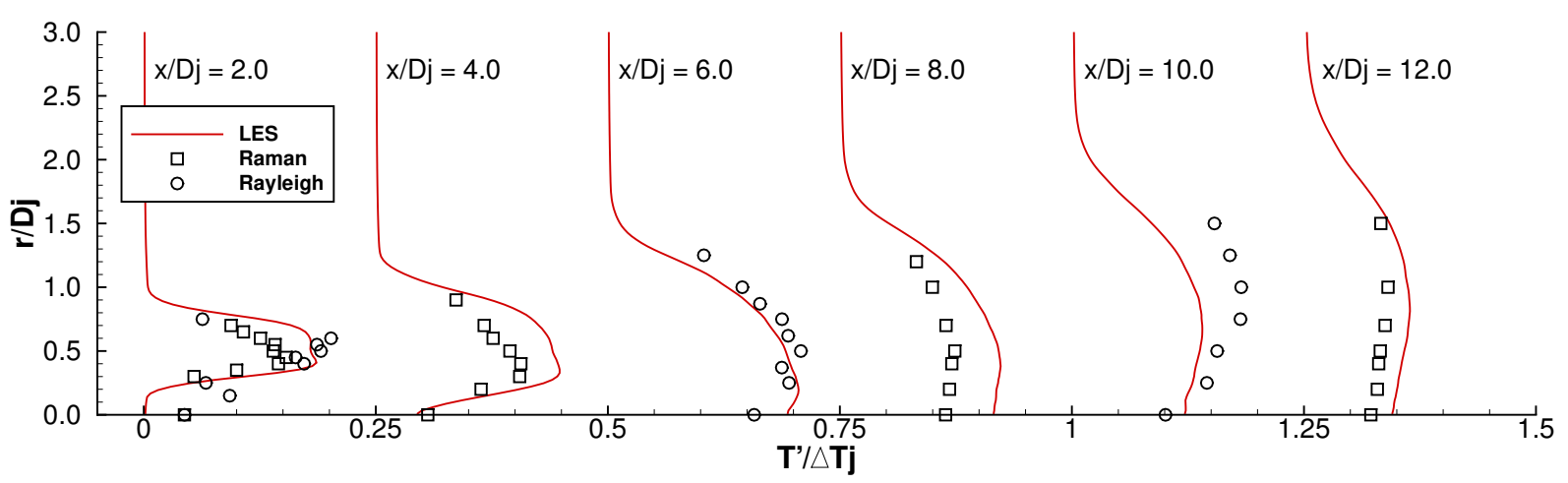

(b) SP 23

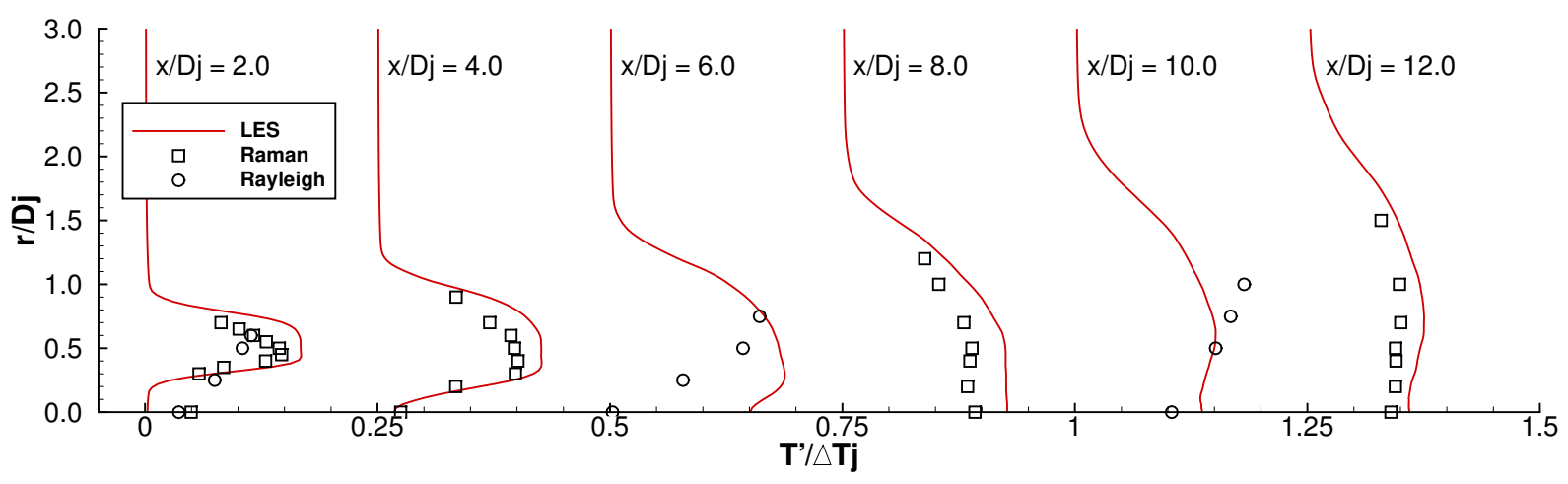

(c) SP 27

Figure 11: Radial profiles of the fluctuation temperature, $T^{\prime} / \Delta T_{j}$. Each profile shifted 0.25 units. 


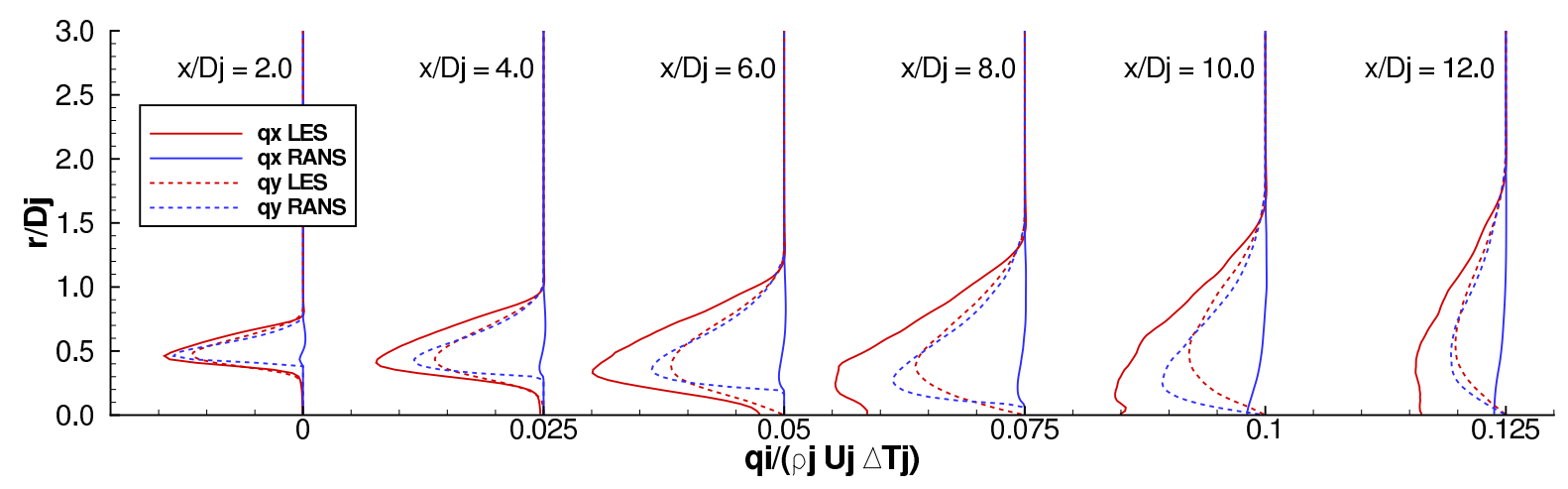

(a) SP 3

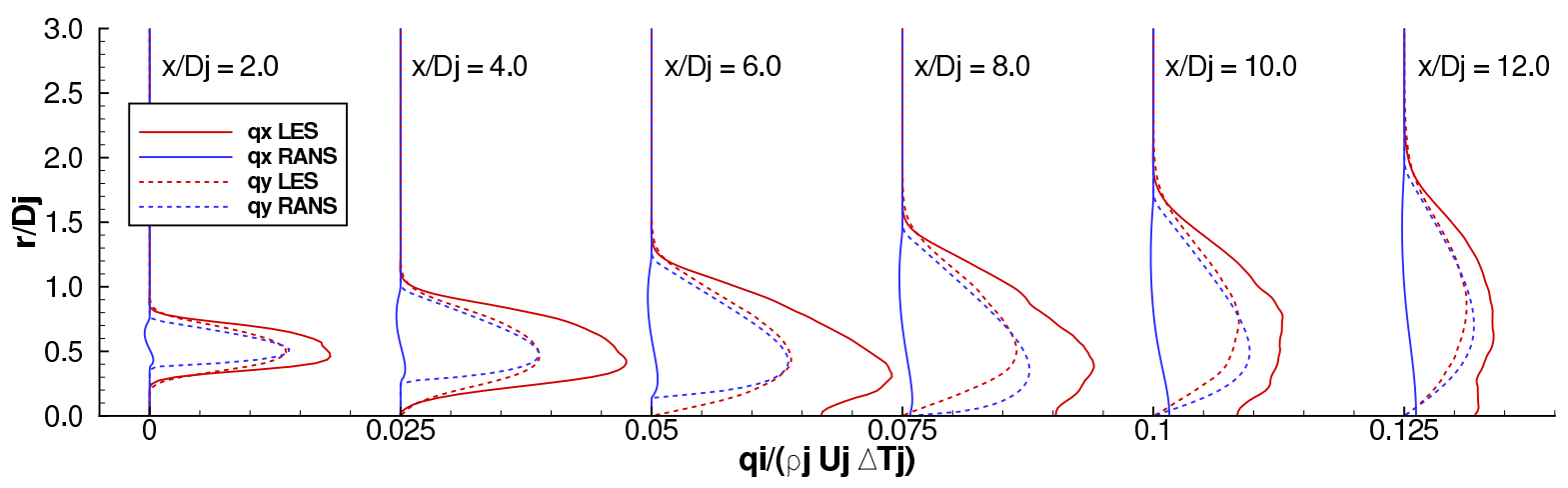

(b) SP 23

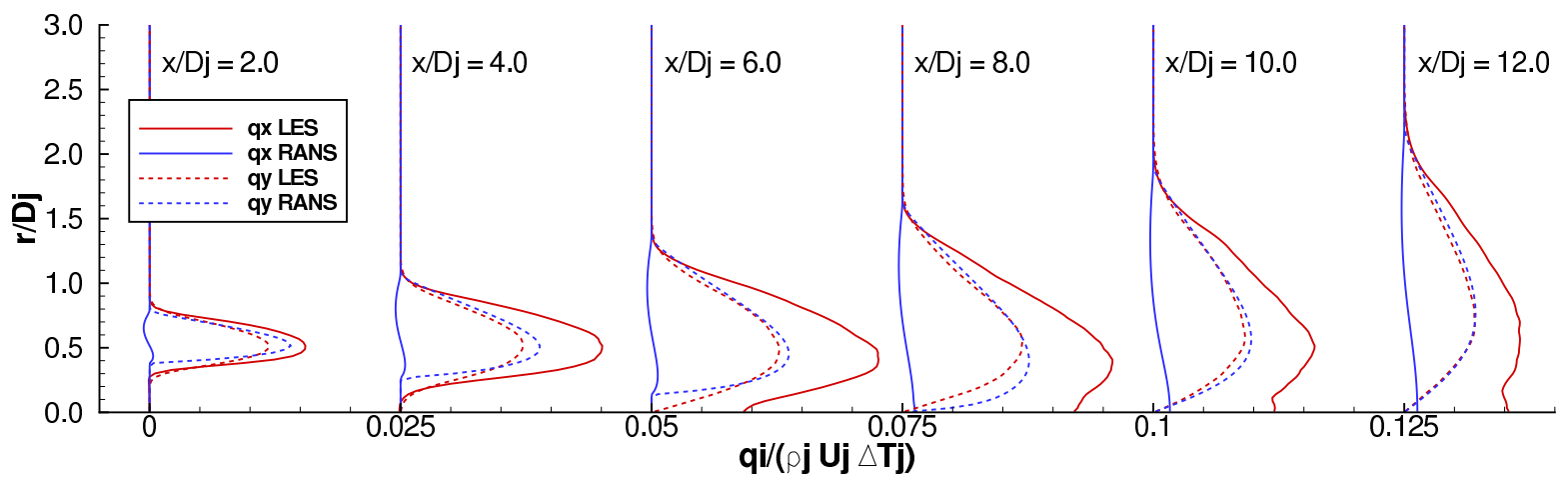

(c) SP 27

Figure 12: Radial profiles of the axial and radial turbulent heat flux, $q_{x}^{T} /\left(\rho_{j} U_{j} \Delta T_{j}\right) \& q_{r}^{T} /\left(\rho_{j} U_{j} \Delta T_{j}\right)$. Each profile shifted 0.025 units. 


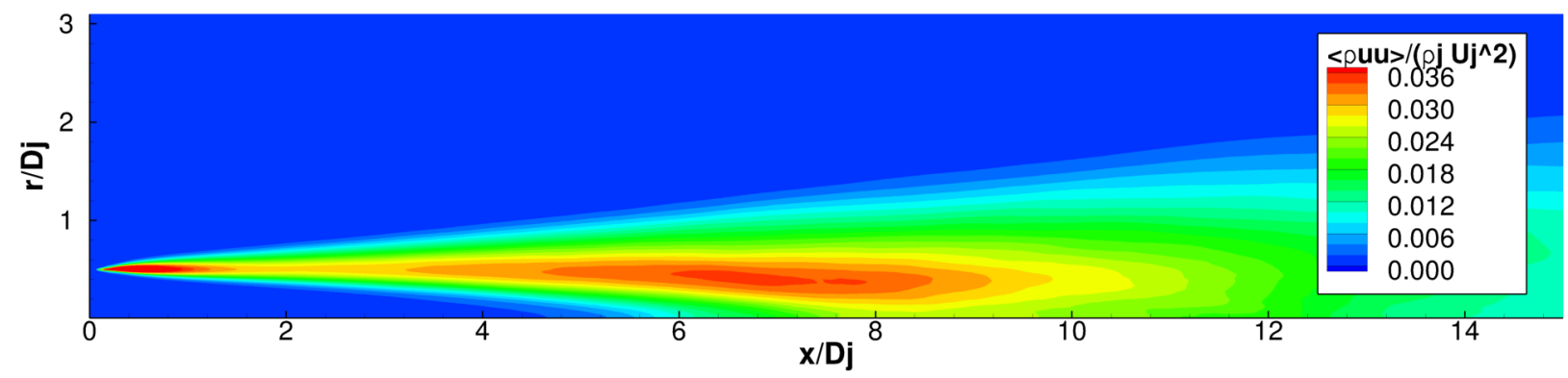

(a) normalized axial Reynolds normal stress, $\overline{\rho u^{\prime} u^{\prime}} /\left(\rho_{j} U_{j}^{2}\right)$

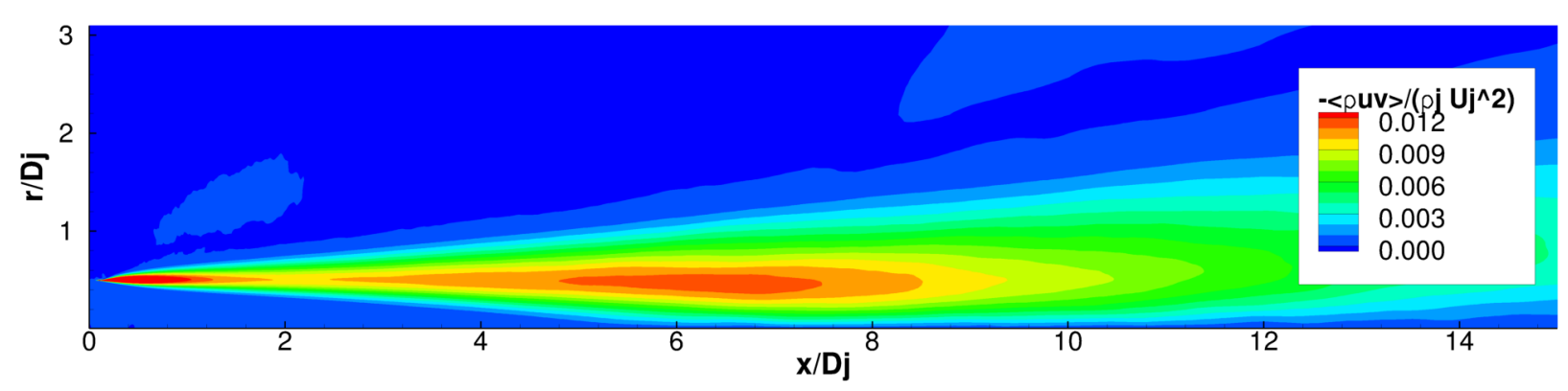

(b) normalized Reynolds shear stress, $-\overline{\rho u^{\prime} v^{\prime}} /\left(\rho_{j} U_{j}^{2}\right)$

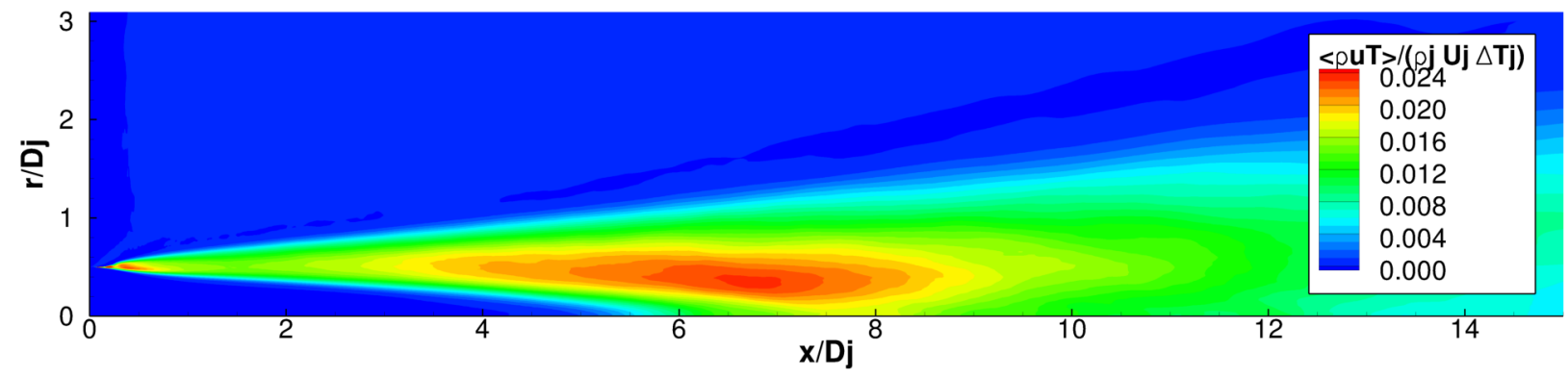

(c) normalized axial turbulent heat flux, $\overline{\rho u^{\prime} T^{\prime}} /\left(\rho_{j} U_{j} \Delta T_{j}\right)$

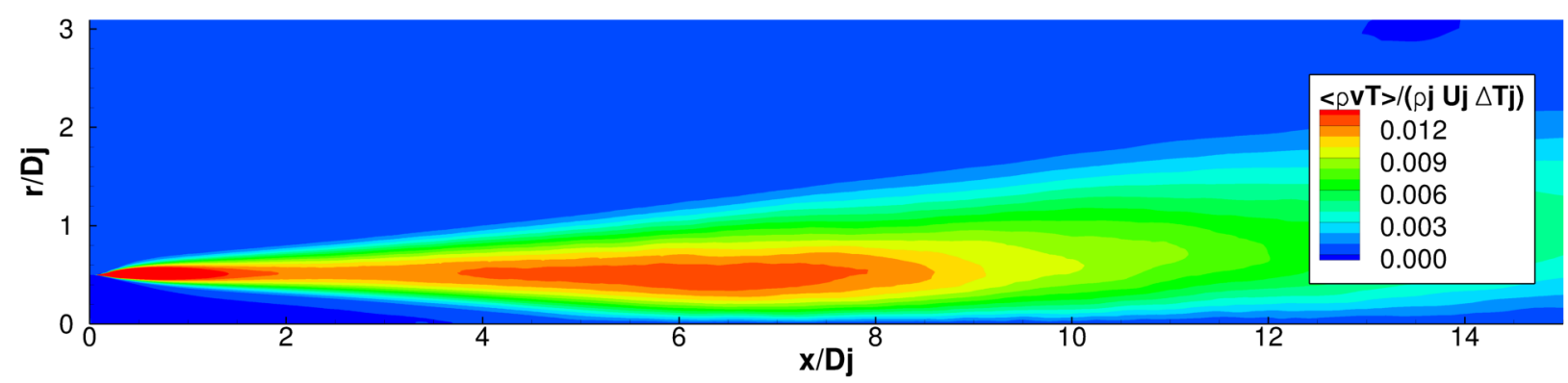

(d) normalized radial turbulent heat flux, $\overline{\rho v^{\prime} T^{\prime}} /\left(\rho_{j} U_{j} \Delta T_{j}\right)$

Figure 13: SP 27, comparison of Reynolds stress and turbulent heat flux contours 


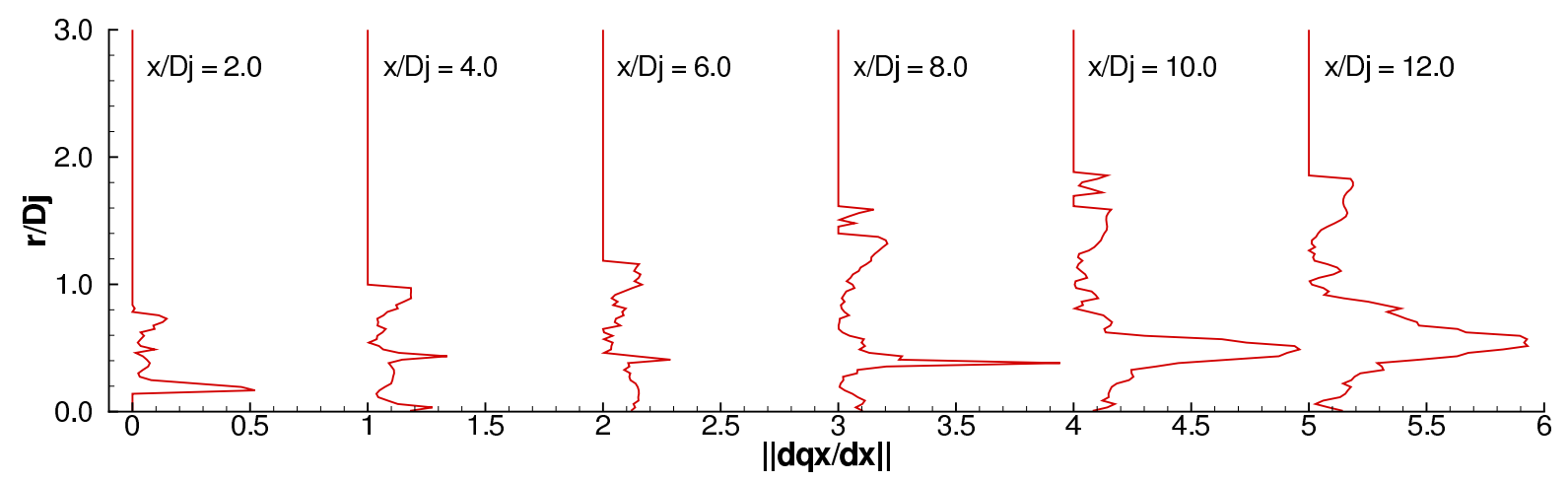

(a) SP 3

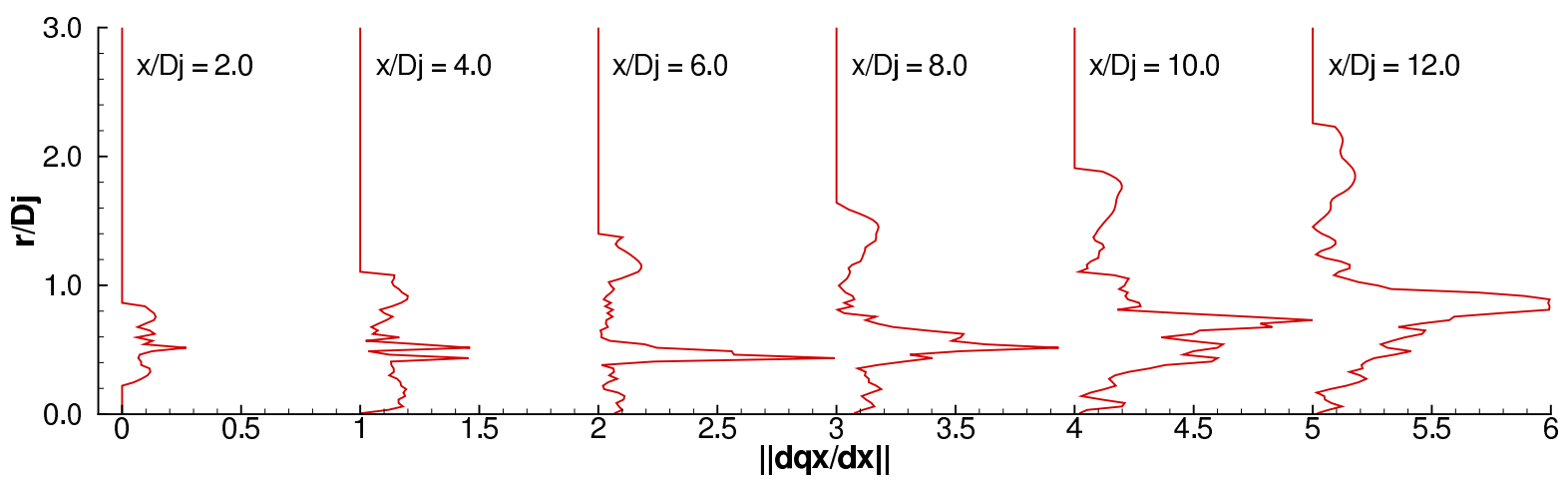

(b) SP 23

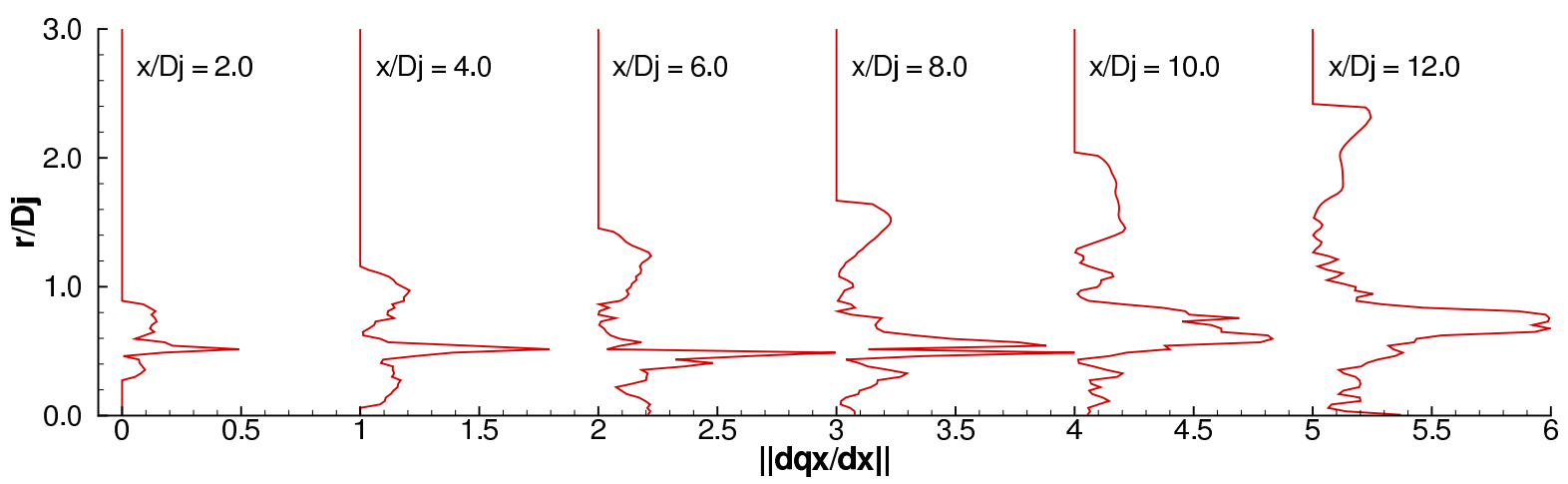

(c) SP 27

Figure 14: Profiles of the normalized magnitude of the axial turbulent heat flux term, $\left\|\partial q_{x}^{T} / \partial x\right\|$ (eqn. 10). Each profile shifted 1 unit. 


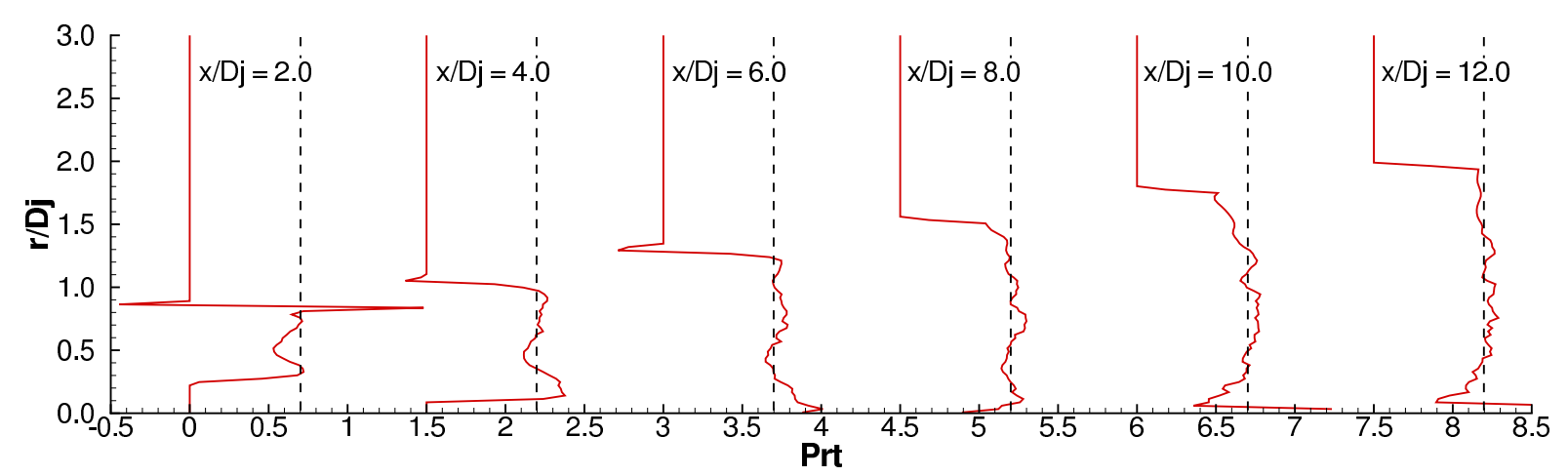

(a) SP 3

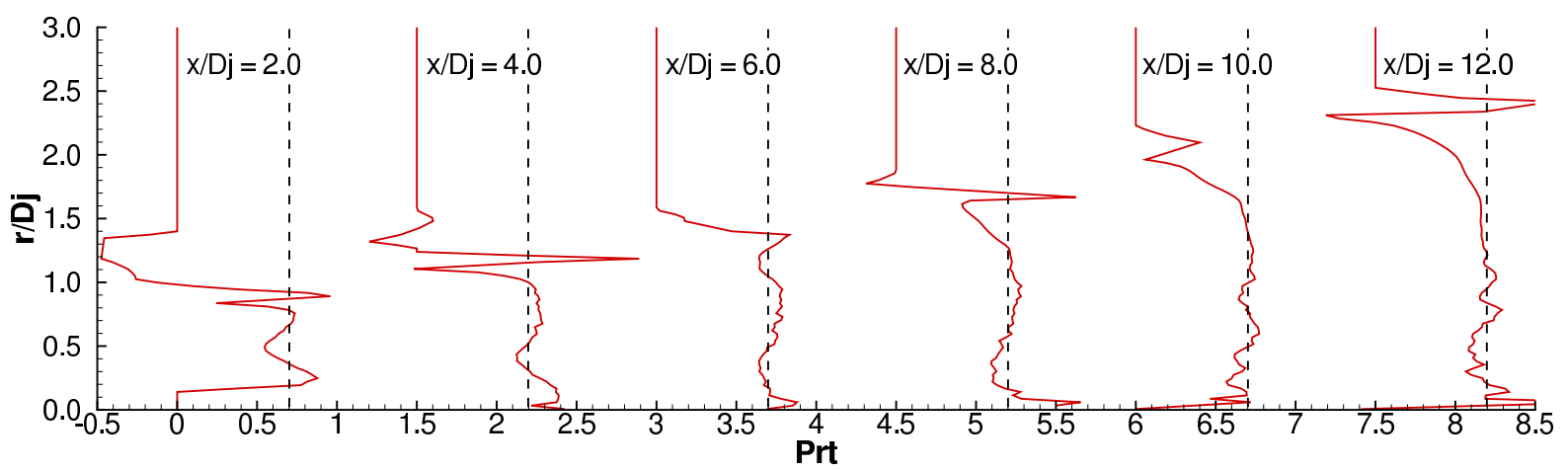

(b) SP 23

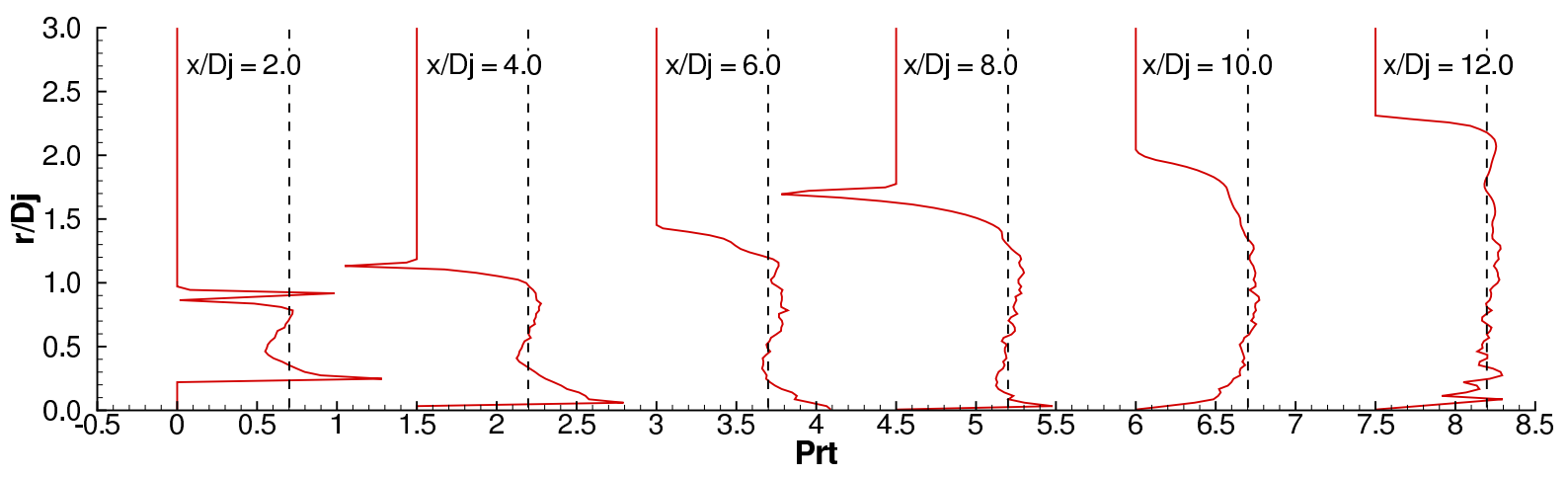

(c) SP 27

Figure 15: Radial profiles of the turbulent Prandtl number, $P r_{t}$. Each profile shifted 1.5 units. Dashed line represents a value of 0.7 . 